\title{
DIGIT survey of far-infrared lines from protoplanetary disks
}

\section{I. [O I], [C II], $\mathrm{OH}, \mathrm{H}_{2} \mathrm{O}$, and $\mathrm{CH}^{+\star, \star \star}$}

\author{
D. Fedele ${ }^{1}$, S. Bruderer ${ }^{1}$, E. F. van Dishoeck ${ }^{1,2}$, J. Carr ${ }^{3}$, G. J. Herczeg ${ }^{4}$, C. Salyk ${ }^{5}$, N. J. Evans II ${ }^{6}$, J. Bouwman ${ }^{7}$, \\ G. Meeus ${ }^{8}$, Th. Henning ${ }^{7}$, J. Green ${ }^{6}$, J. R. Najita ${ }^{5}$, and M. Güdel ${ }^{9}$ \\ 1 Max Planck Institut für Extraterrestrische Physik, Giessenbachstrasse 1, 85748 Garching, Germany \\ e-mail: fedele@mpe.mpg.de \\ 2 Leiden Observatory, Leiden University, PO Box 9513, 2300 RA Leiden, The Netherlands \\ 3 Naval Research Laboratory, Code 7211, Washington, DC 20375, USA \\ 4 Kavli Institute for Astronomy and Astrophysics, Yi He Yuan Lu 5, 100871, Beijing, PR China \\ 5 National Optical Astronomy Observatory, 950 N. Cherry Avenue, Tucson, AZ 85719, USA \\ 6 University of Texas at Austin, Department of Astronomy, 2515 Speedway, Stop C1400, Austin TX 78712-1205, USA \\ 7 Max Planck Institute for Astronomy, Königstuhl 17, 69117 Heidelberg, Germany \\ 8 Universidad Autónoma de Madrid, Dpt. Física Teórica, Campus Cantoblanco, Spain \\ 9 Universität Wien, Dr--Karl-Lueger-Ring 1, 1010 Wien, Austria
}

Received 16 January 2013 / Accepted 26 July 2013

\section{ABSTRACT}

\begin{abstract}
We present far-infrared $(50-200 \mu \mathrm{m})$ spectroscopic observations of young pre-main-sequence stars taken with Herschel/PACS as part of the DIGIT key project. The sample includes 16 Herbig AeBe and $4 \mathrm{~T}$ Tauri sources observed in SED mode covering the entire spectral range. An additional 6 Herbig AeBe and $4 \mathrm{~T}$ Tauri systems have been observed in SED mode with a limited spectral coverage. Multiple atomic fine structure and molecular lines are detected at the source position: [O I], [C II], $\mathrm{CO}, \mathrm{OH}, \mathrm{H}_{2} \mathrm{O}, \mathrm{CH}^{+}$. The most common feature is the [O I] $63 \mu \mathrm{m}$ line detected in almost all of the sources, followed by $\mathrm{OH}$. In contrast with $\mathrm{CO}, \mathrm{OH}$ is detected toward both Herbig AeBe groups (flared and non-flared sources). An isothermal LTE slab model fit to the OH lines indicates column densities of $10^{13}<N_{\mathrm{OH}}<10^{16} \mathrm{~cm}^{-2}$, emitting radii $15<r<100 \mathrm{AU}$ and excitation temperatures $100<T_{\text {ex }}<400 \mathrm{~K}$. We used the non-LTE code RADEX to verify the LTE assumption. High gas densities $\left(n \geq 10^{10} \mathrm{~cm}^{-3}\right)$ are needed to reproduce the observations. The $\mathrm{OH}$ emission thus comes from a warm layer in the disk at intermediate stellar distances. Warm $\mathrm{H}_{2} \mathrm{O}$ emission is detected through multiple lines toward the T Tauri systems AS 205, DG Tau, S CrA and RNO 90 and three Herbig AeBe systems HD 104237, HD 142527, HD 163296 (through line stacking). Overall, Herbig AeBe sources have higher $\mathrm{OH} / \mathrm{H}_{2} \mathrm{O}$ abundance ratios across the disk than do T Tauri disks, from near- to far-infrared wavelengths. Far-infrared $\mathrm{CH}^{+}$emission is detected toward $\mathrm{HD} 100546$ and HD 97048. The slab model suggests moderate excitation $\left(T_{\mathrm{ex}} \sim 100 \mathrm{~K}\right)$ and compact $(r \sim 60 \mathrm{AU})$ emission in the case of HD 100546. Off-source [O I] emission is detected toward DG Tau, whose origin is likely the outflow associated with this source. The [C II] emission is spatially extended in all sources where the line is detected. This suggests that not all [C II] emission is associated with the disk and that there is a substantial contribution from diffuse material around the young stars. The flux ratios of the atomic fine structure lines ([O I] $63 \mu \mathrm{m},[\mathrm{OI}] 145 \mu \mathrm{m},[\mathrm{CII}])$ are analyzed with PDR models and require high gas density $\left(n \gtrsim 10^{5} \mathrm{~cm}^{-3}\right)$ and high UV fluxes $\left(G_{\mathrm{o}} \sim 10^{3}-10^{7}\right)$, consistent with a disk origin for the oxygen lines for most of the sources.
\end{abstract}

Key words. stars: variables: T Tauri, Herbig Ae/Be - astrochemistry - protoplanetary disks

\section{Introduction}

Far-infrared (Far-IR) spectroscopic observations of young premain-sequence stars have the potential to reveal the gas and dust composition of protoplanetary disks in regions not probed at any other wavelengths (e.g., van Dishoeck 2004; Lorenzetti 2005; Henning et al. 2010). The atomic and molecular transitions in the far-IR regime $(50-200 \mu \mathrm{m})$ span a large range in upper energy level (from a few $10 \mathrm{~K}$ to a few $10^{3} \mathrm{~K}$ ) and are sensitive to the warm (a few $10^{2} \mathrm{~K}$ ) upper layers of the disk $\left(n<10^{8} \mathrm{~cm}^{-3}\right)$. For a disk irradiated by UV and/or X-rays

\footnotetext{
* Herschel is an ESA space observatory with science instruments provided by European-led Principal Investigator consortia and with important participation from NASA.

$\star \star$ Appendices are available in electronic form at http://www. aanda.org
}

from the pre-main-sequence star, these conditions are found at intermediate distances from the central star $(r \gtrsim 10 \mathrm{AU})$ (e.g., Kamp \& Dullemond 2004; Bruderer et al. 2012). Observations of lines of multiple species provide a wealth of information that allow us to (1) determine the physical properties of the gas such as excitation temperature, column density, emitting radii (and in some cases the total gas density); (2) constrain the excitation mechanism (e.g., collisions, UV fluorescence, IR pumping); and (3) address the chemical structure of the disk. The far-IR spectrum contains information complementary to that provided by near- and mid-IR observations which are sensitive to the hot $(>1000 \mathrm{~K})$ inner region of the disk $(<\mathrm{a}$ few AU). At the other end of the spectrum, (sub)millimeter spectroscopic observations with ALMA will unveil the physical conditions and chemical composition of the disk midplane at distances $r \gtrsim 10$ AU. The far-IR data probe intermediate disk radii and depths. The 
ultimate goal of these observational campaigns is to use the combined data to address the chemistry and physics of the entire protoplanetary disk from inner to outer edge.

We present here $50-200 \mu \mathrm{m}$ spectra of a sample of protoplanetary disks around Herbig AeBe and T Tauri stars obtained in the context of the "Dust, Ice and Gas in Time" (DIGIT) key program (Sturm et al. 2010). The unprecedented sensitivity of the PACS instrument (Poglitsch et al. 2010) on board the Herschel Space Observatory (Pilbratt et al. 2010) allows for the first time the detection of weak atomic and molecular emission down to a few $10^{-18} \mathrm{~W} \mathrm{~m}^{-2}$. Far-IR spectra of bright Herbig stars have been obtained previously with the Long Wavelength Spectrometer (LWS) on the Infrared Space Observatory (ISO; e.g., Waelkens et al. 1996; Meeus et al. 2001; Giannini et al. 1999; Lorenzetti et al. 1999, 2002; Creech-Eakman et al. 2002). One of the main results has been an empirical classification of the Herbig AeBe systems into two groups based on the ratio of the far- to near-IR (dust) emission (Meeus et al. 2001). Group I sources have a high far- to near-IR emission ratio consistent with a flaring disk geometry while Group II sources have a low flux ratio characteristic of a flat, self-shadowed disk. Grain growth and settling may also play a role (e.g. Acke et al. 2009). One question to be addressed here is to what extent the far-IR gasphase lines reflect this dichotomy in disk structure.

The near-IR spectra of Herbig AeBe disks are characterized by several ro-vibrational lines of CO (e.g. Brittain et al. 2003; Blake \& Boogert 2004; van der Plas et al. 2009; Salyk et al. 2011a) and OH (Mandell et al. 2008; Fedele et al. 2011; Doppmann et al. 2011; Liskowsky et al. 2012). At mid-IR wavelengths the spectra of Herbig AeBe disks are dominated by dust emission and only very few Herbig sources show molecular emission (Pontoppidan et al. 2010; Salyk et al. 2011b). The optical forbidden oxygen lines are common in Herbig AeBe spectra (e.g. Acke \& van den Ancker 2004) and are found to come from the disk atmosphere close to the star $(<10$ AU, e.g., Fedele et al. 2008; van der Plas et al. 2008). In contrast, the emission from $\mathrm{T}$ Tauri systems is characterized by a rich molecular spectrum from near- to mid-IR wavelengths. The inventory of molecular species detected in T Tauri sources in the infrared includes: $\mathrm{CO}$ (e.g. Najita et al. 2003), $\mathrm{OH}$ and $\mathrm{H}_{2} \mathrm{O}$ (e.g., Carr et al. 2004; Salyk et al. 2008), $\mathrm{HCN}$ and $\mathrm{C}_{2} \mathrm{H}_{2}$ (e.g., Carr \& Najita 2008, 2011; Pascucci et al. 2009; Mandell et al. 2012) and, finally $\mathrm{CO}_{2}$ (Pontoppidan et al. 2010). Are Herbig sources also different from $\mathrm{T}$ Tauri sources at far-IR wavelengths?

In this paper we report on the detection of far-IR atomic fine structure lines ([O I] and [C II]) and molecular lines $\left(\mathrm{OH}, \mathrm{H}_{2} \mathrm{O}\right.$, $\mathrm{CH}^{+}$). The analysis of far-IR CO lines is reported in Meeus et al. (2013, hereafter Paper II). This survey over the full PACS wavelength range complements GASPS (Meeus et al. 2012) which targeted specific lines.

\section{Observations and data reduction}

\subsection{Sample}

The sources were selected primarily on their far-IR fluxes such that a $S / N \approx 100$ could be reached on the continuum within $5 \mathrm{~h}$ of integration time. The Herbig AeBe sources in this sample have spectral type between F4 to B9 and are not embedded in large molecular clouds. They have been studied previously at mid-IR wavelengths by Spitzer (Juhász et al. 2010) and the selected sample contains mostly nearby and low-luminosity sources. The T Tauri stars consist of an inhomogeneous sample of bright sources with $\mathrm{K}-\mathrm{G}$ spectral type. AS 205, S CrA, and RU Lup are heavily veiled sources, with CO line profiles suggesting the presence of a disk wind (Bast et al. 2011; Pontoppidan et al. 2011). DG Tau is associated with an outflow that can contribute to the observed emission. In addition RU Lup has evidence for a jet (Güdel et al. 2010). Table 1 provides the parameters of the sample. For the Herbig AeBe sources, the disk group is also indicated: group I sources have flared disk while group II sources have flat disks, in the classification of Meeus et al. (2001).

The focus in this paper is on the Herbig sample, but the data on T Tauri sources are reported for completeness and to allow a comparison with the Herbig sample in a consistent way. More details about the sample are given in Paper II.

\subsection{Observational details}

PACS is an array of $5 \times 5$ spaxels $^{1}$, with spectral energy distribution (SED) each spaxel covering 9!.4×9!'4. The instrument is diffraction limited only at $\lambda<110 \mu \mathrm{m}$. The targets were observed in spectral energy distribution (SED) mode with two settings in order to cover the spectral range $51-220 \mu \mathrm{m}$ (B2A, 51-73 $\mu \mathrm{m}$, short R1, 100-145 $\mu \mathrm{m}$ and B2B, 70-105 $\mu \mathrm{m}$ long $\mathrm{R} 1,140-220 \mu \mathrm{m})$. The spectral resolving power is $R=$ $\lambda / \Delta \lambda \sim 1000$, increasing to 3000 at the shortest wavelengths. A second sample of targets was observed with a limited spectral range (B2A, 60-75 $\mu \mathrm{m}$; short $\mathrm{R} 1,120-143 \mu \mathrm{m}$ ) centered at the position of the forsterite emission but including some specific lines. The observations were carried out in chopping/nodding mode with a chopping throw of $6^{\prime}$. The observation log and parameters of the sample are presented in Table 1.

The data have been reduced with HIPE 8.0.2489 with standard calibration files from level 0 to level 2 (see Green et al. 2013). The two nod positions were reduced separately (oversampling factor $=3$ ) and averaged after a flat-field correction. In the case of HD 100546, which was observed in a different mode during the science demonstration phase, we used an oversampling factor equal to 1 . The spectra are extracted from the central spaxel to optimize the signal-to-noise ratio $(\mathrm{S} / \mathrm{N})$. To flux calibrate the spectra we performed the following steps: 1) correct for flux loss by means of a PSF-loss correction function provided by HIPE; 2) scale to PACS photometry (whenever available); 3) matching spectral modules. Step 1 is valid for objects well centered in the central spaxel. In the case of mispointed observations we extracted the total flux (all 25 spaxels) to recover the flux loss. In this case we fitted a 3rd-order polynomial to two spectra (central spaxel and 25 spaxels). The correction factor is the ratio between the two fits. The mispointed sources are: AB Aur, HD 97048, HD 169142, HD 142666. The regions affected by spectral leakage (B2B 95-105 $\mu \mathrm{m}$ and R1 $190-220 \mu \mathrm{m})$ are excluded from this procedure. Based on a statistical analysis, the PACS SED fluxes agree with PACS photometry to within 5-10\%. For this reason we assign an uncertainty of $10 \%$ to the PACS SED fluxes of sources without PACS photometry available.

The line fluxes are measured by fitting a Gaussian function and the uncertainty $(\sigma)$ is given by the product $\operatorname{STD}_{\mathrm{F}} \delta \lambda \sqrt{N_{\text {bin }}}$, where $\mathrm{STD}_{\mathrm{F}}$ is the standard deviation of the (local) spectrum ( $\left.\mathrm{W} \mathrm{m}^{-2} \mu \mathrm{m}^{-1}\right), \delta \lambda$ is the wavelength spacing of the bins $(\mu \mathrm{m})$ and $N_{\text {bin }}$ is the width of the line in spectral bins ( 5 for all lines).

A spaxel is a spatial sampling element of the PACS integral field unit. 
D. Fedele et al.: DIGIT survey of far-infrared lines from protoplanetary disks. I.

Table 1. Properties of the program stars and PACS observation log.

\begin{tabular}{|c|c|c|c|c|c|c|c|}
\hline Star & $\begin{array}{c}\mathrm{RA} \\
(\mathrm{J} 2000)\end{array}$ & $\begin{array}{c}\text { Dec } \\
(\mathrm{J} 2000)\end{array}$ & Sp. Type & $\begin{array}{c}\text { Distance } \\
{[\mathrm{pc}]}\end{array}$ & Group & $\begin{array}{c}\text { Obsid } \\
(1342+)\end{array}$ & Obs. date \\
\hline AB Aur & 045545.8 & +303304.3 & A0 & $140 \pm 15^{a}$ & I & $217842 / 3$ & $2011 / 04 / 04$ \\
\hline HD 35187 & 052401.2 & +245737.6 & $\mathrm{~A} 2+\mathrm{A} 7$ & $114 \pm 24^{a}$ & II & $217846^{\dagger}$ & $2011 / 04 / 04$ \\
\hline HD 36112 & 053027.5 & +251957.0 & A5 & $280 \pm 55^{a}$ & I & $228247 / 8$ & 2011/09/07 \\
\hline HD 38120 & 054311.9 & -045949.9 & B9 & $480 \pm 175^{a}$ & I & $226212 / 3$ & $2011 / 08 / 15$ \\
\hline HD 50138 & 065133.4 & -065759.5 & B9 & $390 \pm 70^{a}$ & II & $206991 / 2$ & $2010 / 10 / 23$ \\
\hline HD 97048 & 110803.3 & -773917.4 & A0 & $160 \pm 15^{a}$ & I & $199412 / 3$ & 2010/06/30 \\
\hline HD 98922 & 112231.7 & -532211.5 & B9 & $1150_{-355}^{+935 a}$ & II & $210385^{\ddagger}$ & $2010 / 11 / 27$ \\
\hline HD 100453 & 113305.6 & -541928.5 & A9 & $122 \pm 10^{a}$ & I & $211695 / 6$ & $2010 / 12 / 25$ \\
\hline HD 100546 & 113325.4 & -701141.2 & B9 & $97 \pm 4^{a}$ & I & $188038 / 7$ & 2009/12/11 \\
\hline HD 104237 & 120005.1 & -781134.6 & A4 & $116 \pm 5^{a}$ & II & $207819 / 20$ & 2010/11/03 \\
\hline HD 135344 B & 151548.4 & -370916.0 & F4 & $140 \pm 27^{b}$ & I & $213921 / 2$ & 2011/02/07 \\
\hline HD 139614 & 154046.4 & -422953.5 & A7 & $140 \pm 5^{c}$ & I & $215683 / 4$ & 2011/03/10 \\
\hline HD 141569 A & 154957.8 & -035516.3 & A0 & $116 \pm 7^{a}$ & II & $213913^{\ddagger}$ & 2011/02/06 \\
\hline HD 142527 & 155641.9 & -421923.2 & F6 & $230 \pm 50^{a}$ & I & $216174 / 5$ & 2011/03/16 \\
\hline HD 142666 & 155640.0 & -220140.0 & A8 & $145 \pm 5^{c}$ & II & $213916^{\ddagger}$ & 2011/02/06 \\
\hline HD 144432 & 160657.9 & -274309.7 & A9 & $160 \pm 25^{a}$ & II & $213919^{\ddagger}$ & 2011/02/07 \\
\hline HD 144668 & 160834.3 & -390618.3 & A1/A2 & $160 \pm 15^{a}$ & II & $215641 / 2$ & 2011/03/08 \\
\hline Oph IRS 48 & 162737.2 & -243035.0 & A0 & $120 \pm 4^{d}$ & I & $227069 / 70$ & $2011 / 08 / 22$ \\
\hline HD 150193 & 164017.9 & -235345.2 & A2 & $203 \pm 40^{a}$ & II & $227068^{\ddagger}$ & $2011 / 08 / 22$ \\
\hline HD 163296 & 175621.3 & -215721.9 & A1 & $120 \pm 10^{a}$ & II & $217819 / 20$ & 2011/04/03 \\
\hline HD 169142 & 182429.8 & -294649.3 & A8 & $145 \pm 5^{c}$ & I & $206987 / 8$ & $2010 / 10 / 23$ \\
\hline HD 179218 & 191111.3 & +154715.6 & A0 & $255 \pm 40^{a}$ & I & $208884 / 5$ & 2010/11/12 \\
\hline DG Tau & 042704.7 & +260616.3 & K5 & $140^{e}$ & & $225730 / 1$ & $2011 / 11 / 15$ \\
\hline HT Lup & 154512.9 & -341730.6 & K2 & $120 \pm 35^{a}$ & & $213920^{\ddagger}$ & $2011 / 11 / 17$ \\
\hline RU Lup & 155642.3 & -374915.5 & G5 & $120 \pm 35^{a}$ & & $215682^{\ddagger}$ & $2011 / 03 / 10$ \\
\hline RY Lup & 155928.4 & -402151.2 & K4 & $120 \pm 35^{a}$ & & 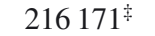 & $2011 / 03 / 16$ \\
\hline AS 205 & 161131.4 & -183824.5 & K5 & $125^{f}$ & & $215737 / 8$ & 2011/11/18 \\
\hline EM* SR 21 & 162710.3 & -241912.5 & G3 & $120 \pm 4^{f}$ & & $227209 / 10$ & 2011/08/14 \\
\hline RNO 90 & 163409.2 & -154816.8 & G5 & $125 \pm 4^{f}$ & & $228206^{\ddagger}$ & 2011/09/06 \\
\hline S Cra & 190108.6 & -365719.9 & $\mathrm{~K} 3+\mathrm{M} 0$ & $129 \pm 11^{g}$ & & $207809 / 10$ & $2010 / 11 / 02$ \\
\hline
\end{tabular}

Notes. ${ }^{(a)}$ van Leeuwen (2007); ${ }^{(b)}$ Müller et al. (2011); ${ }^{(c)}$ Acke \& van den Ancker (2004), and references therein; ${ }^{(d)}$ Loinard et al. (2008); ${ }^{(e)}$ Kenyon et al. (2008); ${ }^{(f)}$ Pontoppidan et al. $(2011)$, and references therein; ${ }^{(g)}$ Neuhäuser \& Forbrich $(2008)$; ${ }^{(\dagger)}$ spectral coverage $=50-73 \mu$ m,

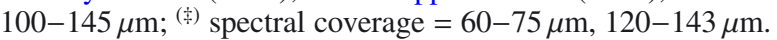

\section{Results}

\subsection{Overview}

An overview of the detected atomic and molecular species is shown in Table 2. Figure A.1 shows the continuum normalized PACS spectrum of a T Tauri star (AS 205) and of an Herbig AeBe star (HD 97048). Figures A.2 and A.3 show a portion of the PACS spectrum (continuum normalized) of selected sources. The strongest and most common feature is the [O I] $63 \mu \mathrm{m}$ line, seen in all but 4 sources. The [O I] $145 \mu \mathrm{m}$ and [C II] $157 \mu \mathrm{m}$ lines are also detected, usually in the same sources, although the detection rate is much lower for these two lines. Four molecular species are seen: $\mathrm{CO}, \mathrm{OH}, \mathrm{H}_{2} \mathrm{O}$ and $\mathrm{CH}^{+}$. Line fluxes are reported in Tables 3-5 and B.1. The CO lines are presented in Paper II. After [O I] $63 \mu \mathrm{m}, \mathrm{OH}$ emission is the most common feature, detected in $40 \%$ of the sources with full spectral coverage.

We searched for other species such as [N II], HD and $\mathrm{OH}^{+}$. The HD $J=1-0$ line at $112 \mu \mathrm{m}$ has been detected toward TW Hya with a flux of $6.3( \pm 0.7) \times 10^{-18} \mathrm{~W} \mathrm{~m}^{-2}$ after deep integration (Bergin et al. 2013). None of the sources analyzed here shows evidence of [N II], $\mathrm{HD}$ or $\mathrm{OH}^{+}$emission with $3 \sigma$ upper limits of the order of $1-2 \times 10^{-17} \mathrm{~W} \mathrm{~m}^{-2}$ for most of the sources. Typical upper limits in different parts of individual PACS spectra can be derived from upper limits on nearby $\mathrm{OH}$ lines in Table B.1.

\section{2. $[\mathrm{O} I]$}

The [O I] $63 \mu \mathrm{m}$ line is the most common and strongest feature detected throughout the whole sample. The only sources in which the line is not detected are HD 142666, HD 144432 and SR 21. The line flux ranges from $10^{-17}$ to $10^{-15} \mathrm{~W} \mathrm{~m}^{-2}$. The [O I] $145 \mu \mathrm{m}$ line is detected in 7 (out of 16) HAeBe stars and in 3 (out of 4) T Tauri stars. In both cases, the spatial distribution of the line emission in the PACS array follows the shape of the PSF and the emission is not spatially extended. Figures A.2 and A.3 show the [O I] spectra for a selected sample.

Excess emission is detected outside the central spaxel toward DG Tau (see Appendix D) in agreement with Podio et al. (2012). In this case, the fluxes of the $[\mathrm{OI}] 63 \mu \mathrm{m}$ lines are lower from those reported by Podio et al. (2012) who computed the line fluxes by adding all the spaxels (thus including off-source emission). The [O I] $63 \mu \mathrm{m}$ line flux of DG Tau in Table 3 refers to the on-source position only (spectrum extracted from the central spaxel and corrected for PSF-loss, see Appendix D).

\section{3. $\mathrm{OH}$}

The most common molecular species detected in the PACS spectra is the hydroxyl radical, $\mathrm{OH}$. Six $\mathrm{OH}$ doublets with upper energy levels up to $875 \mathrm{~K}$ are found including a cross-ladder transition ${ }^{2} \Pi_{1 / 2}-{ }^{2} \Pi_{3 / 2} J=1 / 2-3 / 2$ at $79 \mu \mathrm{m}$. No spatially extended 
Table 2. Overview of detected species.

\begin{tabular}{lccccccc}
\hline \hline Star & \multicolumn{2}{c}{ [O I] } & [C II] & $\mathrm{CO}^{a}$ & $\mathrm{OH}$ & $\mathrm{H}_{2} \mathrm{O}$ & $\mathrm{CH}^{+}$ \\
& $63 \mu \mathrm{m}$ & $145 \mu \mathrm{m}$ & & & & & \\
\hline AB Aur & $\mathrm{Y}$ & $\mathrm{Y}$ & $\mathrm{Y}$ & $\mathrm{Y}$ & $\mathrm{Y}$ & $\mathrm{n}$ & $\mathrm{n}$ \\
HD 35187 & $\mathrm{Y}$ & $\mathrm{n}^{b}$ & $\mathrm{n}^{b}$ & $\mathrm{n}$ & $\mathrm{n}$ & $\mathrm{n}$ & $\mathrm{n}$ \\
HD 36112 & $\mathrm{Y}$ & $\mathrm{n}$ & $\mathrm{n}$ & $\mathrm{Y}$ & $\mathrm{Y}$ & $\mathrm{n}$ & $\mathrm{n}$ \\
HD 38120 & $\mathrm{Y}$ & $\mathrm{Y}$ & $\mathrm{Y}$ & $\mathrm{n}$ & $?$ & $\mathrm{n}$ & $\mathrm{n}$ \\
HD 50138 & $\mathrm{Y}$ & $\mathrm{Y}$ & $\mathrm{Y}$ & $\mathrm{n}$ & $\mathrm{Y}$ & $\mathrm{n}$ & $\mathrm{n}$ \\
HD 97048 & $\mathrm{Y}$ & $\mathrm{Y}$ & $\mathrm{Y}$ & $\mathrm{Y}$ & $\mathrm{Y}$ & $\mathrm{n}$ & $\mathrm{Y}$ \\
HD 98922 & $\mathrm{Y}$ & - & - & $\mathrm{n}$ & $\mathrm{n}$ & $\mathrm{n}$ & $\mathrm{n}$ \\
HD 100453 & $\mathrm{Y}$ & $\mathrm{n}$ & $\mathrm{n}$ & $\mathrm{n}$ & $\mathrm{n}$ & $\mathrm{n}$ & $\mathrm{n}$ \\
HD 100546 & $\mathrm{Y}$ & $\mathrm{Y}$ & $\mathrm{Y}$ & $\mathrm{Y}$ & $\mathrm{Y}$ & $\mathrm{n}$ & $\mathrm{Y}$ \\
HD 104237 & $\mathrm{Y}$ & $\mathrm{n}$ & $\mathrm{n}$ & $\mathrm{n}$ & $\mathrm{Y}$ & $\mathrm{Y}$ & $\mathrm{n}$ \\
HD 135344 B & $\mathrm{Y}$ & $\mathrm{n}$ & $\mathrm{n}$ & $\mathrm{n}$ & $\mathrm{n}$ & $\mathrm{n}$ & $\mathrm{n}$ \\
HD 139614 & $\mathrm{Y}$ & $\mathrm{n}$ & $\mathrm{n}$ & $\mathrm{n}$ & $\mathrm{n}$ & $\mathrm{n}$ & $\mathrm{n}$ \\
HD 141569 & $\mathrm{Y}$ & $\mathrm{Y}^{b}$ & $\mathrm{Y}^{b}$ & $\mathrm{n}$ & $\mathrm{n}$ & $\mathrm{n}$ & $\mathrm{n}$ \\
HD 142527 & $\mathrm{Y}$ & $\mathrm{n}$ & $\mathrm{n}$ & $\mathrm{n}$ & $?$ & $\mathrm{Y}$ & $\mathrm{n}$ \\
HD 142666 & $\mathrm{n}^{c}$ & $\mathrm{n}^{b}$ & $\mathrm{n}^{b}$ & $\mathrm{~N}$ & $?$ & $\mathrm{n}$ & $\mathrm{n}$ \\
HD 144432 & $\mathrm{n}$ & - & - & $\mathrm{n}$ & $\mathrm{n}$ & $\mathrm{n}$ & $\mathrm{n}$ \\
HD 144668 & $\mathrm{Y}$ & $\mathrm{n}$ & $\mathrm{n}$ & $\mathrm{n}$ & $\mathrm{n}$ & $\mathrm{n}$ & $\mathrm{n}$ \\
Oph IRS 48 & $\mathrm{Y}$ & $\mathrm{Y}$ & $\mathrm{Y}$ & $\mathrm{Y}$ & $\mathrm{n}$ & $\mathrm{n}$ & $\mathrm{n}$ \\
HD 150193 & $\mathrm{Y}$ & $\mathrm{n}^{b}$ & $\mathrm{n}^{b}$ & $\mathrm{n}$ & $\mathrm{n}$ & $\mathrm{n}$ & $\mathrm{n}$ \\
HD 163296 & $\mathrm{Y}$ & $\mathrm{n}$ & $\mathrm{n}$ & $\mathrm{n}$ & $\mathrm{Y}$ & $\mathrm{Y}$ & $\mathrm{n}$ \\
HD 169142 & $\mathrm{Y}$ & $\mathrm{n}$ & $\mathrm{n}$ & $\mathrm{n}$ & $\mathrm{n}$ & $\mathrm{n}$ & $\mathrm{n}$ \\
HD 179218 & $\mathrm{Y}$ & $\mathrm{Y}$ & $\mathrm{Y}$ & $\mathrm{n}$ & $\mathrm{n}$ & $\mathrm{n}$ & $\mathrm{n}$ \\
\hline DG Tau & $\mathrm{Y}$ & $\mathrm{Y}$ & $\mathrm{Y}$ & $\mathrm{Y}$ & $\mathrm{Y}$ & $\mathrm{Y}$ & $\mathrm{n}$ \\
HT Lup & $\mathrm{Y}$ & - & - & $\mathrm{n}$ & $\mathrm{n}$ & $\mathrm{n}$ & $\mathrm{n}$ \\
RU Lup & $\mathrm{Y}$ & - & - & $\mathrm{n}$ & $\mathrm{Y}$ & $\mathrm{n}$ & $\mathrm{n}$ \\
RY Lup & $\mathrm{Y}$ & - & - & $\mathrm{n}$ & $\mathrm{n}$ & $\mathrm{n}$ & $\mathrm{n}$ \\
AS 205 & $\mathrm{Y}$ & $\mathrm{Y}$ & $\mathrm{n}$ & $\mathrm{Y}$ & $\mathrm{Y}$ & $\mathrm{Y}$ & $\mathrm{n}$ \\
EM* SR 21 & $\mathrm{n}$ & $\mathrm{n}$ & $\mathrm{Y}$ & $\mathrm{n}$ & $\mathrm{n}$ & $\mathrm{n}$ & $\mathrm{n}$ \\
RNO 90 & $\mathrm{Y}$ & - & - & $\mathrm{n}$ & $\mathrm{Y}$ & $\mathrm{Y}$ & $\mathrm{n}$ \\
S Cra & $\mathrm{Y}$ & $\mathrm{Y}$ & $\mathrm{n}$ & $\mathrm{Y}$ & $\mathrm{Y}$ & $\mathrm{Y}$ & $\mathrm{n}$ \\
\hline & & & & & & & \\
& & & & &
\end{tabular}

Notes. ${ }^{(a)}$ The analysis of the CO lines is presented in Paper II. The symbol "_" means species not observed. ${ }^{(b)}$ Data not available in DIGIT. Line observed by Meeus et al. (2012). ${ }^{(c)}$ Line detected by Meeus et al. (2012).

$\mathrm{OH}$ emission is detected outside the central spaxel of the PACS array. The emission is seen in both Herbig AeBe groups (flared and flat) as well as in T Tauri stars (Figs. A.1-A.3).

\section{4. $\mathrm{H}_{2} \mathrm{O}$}

$\mathrm{H}_{2} \mathrm{O}$ lines are detected toward the T Tauri sources AS 205, DG Tau, and S CrA, including transitions from high-excitation levels $\left(E_{\mathrm{u}} \sim 1000 \mathrm{~K}\right)$. Different transitions are detected in different targets and, interestingly, the strongest lines come from high energy levels in contrast to embedded sources where the strongest lines are from low energy levels (e.g., Herczeg et al. 2012). These differences are likely due to different excitation mechanisms (e.g. collisions, infrared pumping, shocks) and different physical conditions (temperature and column density). The non detection of low-energy lines is further discussed in Sect. 4.3.3. The target with the richest $\mathrm{H}_{2} \mathrm{O}$ spectrum is AS 205 with 10 lines detected. Individual line fluxes are reported in Table 5 together with $3 \sigma$ upper limits to some of the low-energy backbone lines for AS 205. Far-IR $\mathrm{H}_{2} \mathrm{O}$ emission in DG Tau has also been detected by Podio et al. (2012) using PACS. The line fluxes agree within 10-30\% due to different flux calibration. Weak $\mathrm{H}_{2} \mathrm{O}$ emission is also detected toward RNO 90 through
Table 3. [O I] and [C II] line fluxes.

\begin{tabular}{lccc}
\hline \hline Star & {$[\mathrm{O} \mathrm{I}] 63 \mu \mathrm{m}$} & {$[\mathrm{O} \mathrm{I}] 145 \mu \mathrm{m}$} & {$[\mathrm{C} \text { II }]^{a}$} \\
\hline AB Aur & $94.6 \pm 5.2$ & $3.7 \pm 0.7$ & 2.0 \\
HD 35187 & $4.8 \pm 2.0$ & - & - \\
HD 36112 & $5.6 \pm 0.7$ & $<1.1$ & $<1.2$ \\
HD 38120 & $7.6 \pm 0.8$ & $0.7 \pm 0.1$ & 3.3 \\
HD 50138 & $240 \pm 10$ & $6.6 \pm 0.2$ & 7.8 \\
HD 97048 & $136 \pm 5$ & $5.3 \pm 0.5$ & 6.3 \\
HD 98922 & $23.1 \pm 1.2$ & - & - \\
HD 100453 & $10.2 \pm 0.7$ & $<1.2$ & $<1.3$ \\
HD 100546 & $596 \pm 6$ & $21.1 \pm 1.1$ & 17.6 \\
HD 104237 & $7.4 \pm 0.7$ & $<1.5$ & $<1.5$ \\
HD 135344 B & $3.6 \pm 0.5$ & $<1.2$ & $<1.4$ \\
HD 139614 & $3.1 \pm 0.4$ & $<1.2$ & $<1.3$ \\
HD 141569 A & $25.3 \pm 1.5$ & - & - \\
HD 142666 & $<50$ & - & - \\
HD 142527 & $3.6 \pm 0.8$ & $<2.9$ & $<2.8$ \\
HD 144432 & $<5.6$ & - & - \\
HD 144668 & $13.3 \pm 1.0$ & $<0.9$ & $<1.1$ \\
Oph IRS 48 & $30.8 \pm 1.5$ & $2.9 \pm 0.6$ & 1.2 \\
HD 150193 & $3.2 \pm 0.7$ & - & - \\
HD 163296 & $18.2 \pm 0.9$ & $<1.3$ & $<1.3$ \\
HD 169142 & $8.9 \pm 2.0$ & $<2.2$ & $<2.5$ \\
HD 179218 & $17.9 \pm 0.9$ & $0.95 \pm 0.1$ & $0.44^{b}$ \\
\hline DG Tau & $153 \pm 2.0$ & $8.3 \pm 0.4$ & 7.4 \\
HT Lup & $4.0 \pm 0.8$ & - & - \\
RU Lup & $18.9 \pm 1.2$ & - & - \\
RY Lup & $5.0 \pm 2.0$ & - & - \\
AS 205 & $21.5 \pm 1.4$ & $1.6 \pm 0.4$ & $<1.5$ \\
EM* SR 21 & $<5.4$ & $<1.3$ & 0.13 \\
RNO 90 & $12.5 \pm 1.0$ & - & - \\
S Cra & $43.6 \pm 1.3$ & $1.8 \pm 0.5$ & $<1.7$ \\
\hline
\end{tabular}

Notes. Flux unit $10^{-17} \mathrm{~W} \mathrm{~m}^{-2}$. Flux uncertainties refer to $1 \sigma$ error. For non detection the $3 \sigma$ upper limit is given. ${ }^{(a)}$ After subtraction of the extended emission. ${ }^{(b)}[\mathrm{C}$ II] emission is only detected in the central spaxel.

line stacking as shown in Fig. 1 (see below for details of the method).

Herbig AeBe sources show weak or no $\mathrm{H}_{2} \mathrm{O}$ far-IR emission. Weak lines have been reported toward HD 163296 (Fedele et al. 2012; Meeus et al. 2012) and have been confirmed through a stacking analysis. Two other Herbig AeBe stars show hints of $\mathrm{H}_{2} \mathrm{O}$ emission: HD 142527 and HD 104237. The lines are weak, with line fluxes ranging between a few $10^{-18} \mathrm{~W} \mathrm{~m}^{-2}$ and a few $10^{-17} \mathrm{~W} \mathrm{~m}^{-2}$, often below the $3 \sigma$ limit. To confirm the presence of $\mathrm{H}_{2} \mathrm{O}$ emission in these sources, we performed a line stacking analysis as described in detail in Fedele et al. (2012). In brief, the stacking consists in averaging the spectral segments containing $\mathrm{H}_{2} \mathrm{O}$ lines, based on a template of observed $\mathrm{H}_{2} \mathrm{O}$ lines by Herczeg et al. (2012). Spectral bins containing other emission lines ([O I], $\mathrm{OH}, \mathrm{CO}$ and $\mathrm{CH}^{+}$) are masked, and blended $\mathrm{H}_{2} \mathrm{O}$ lines are excluded from the analysis. The stacked $\mathrm{H}_{2} \mathrm{O}$ spectra of HD 163296, HD 142527, HD 104237 and of the T Tauri source RNO 90 are shown in Fig. 1. The false alarm probability, i.e. the probability to detect a signal of equal intensity by stacking random portions of the PACS spectrum, is measured by counting the occurrences of detection in a simulation of 50000 random stackings (after masking the spectral bins containing $\mathrm{H}_{2} \mathrm{O}, \mathrm{OH}, \mathrm{CO}, \mathrm{CH}^{+}$, [O I] and [C II] lines). More details are given in Fedele et al. (2012). The false alarm probability is $0.02 \%$ for HD $142527,0.2 \%$ for HD 104237 and $0.6 \%$ for RNO 90 based on 50000 randomized tests compared to a false 
D. Fedele et al.: DIGIT survey of far-infrared lines from protoplanetary disks. I.

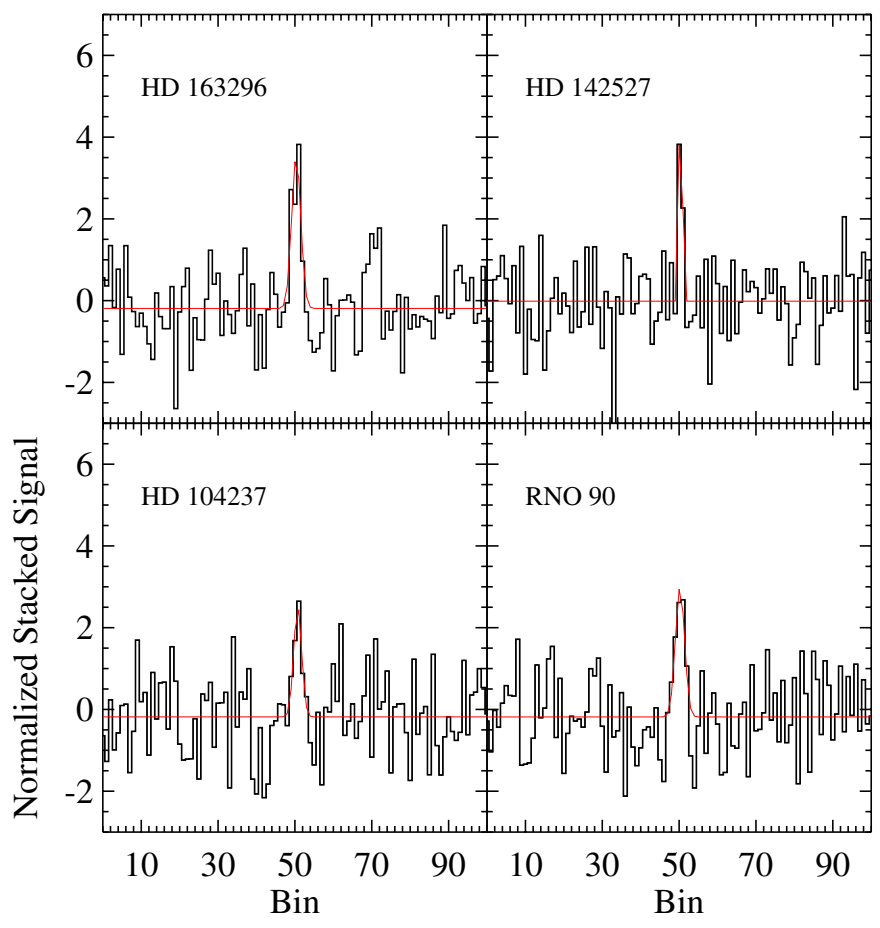

Fig. 1. $\mathrm{H}_{2} \mathrm{O}$ line stacking for the Herbig AeBe sources HD 163296, HD 142527 and HD 104237 and for the T Tauri star RNO 90. The stacked spectrum is divided by the standard deviation of the baseline.

alarm probability of $<0.03 \%$ for HD 163296 . None of the other sources show evidence for the presence of warm $\mathrm{H}_{2} \mathrm{O}$.

Figure 2 shows the average PACS spectrum of the T Tauri and Herbig AeBe sources around $65 \mu \mathrm{m}$. The spectrum of each individual source is continuum subtracted and is divided by the local standard deviation. The source spectra in each category are then summed. The spectrum of HD 100546 was excluded from the Herbig AeBe list because of its lower spectral sampling. These average spectra demonstrate that $\mathrm{OH}$ emission is detected in both classes of objects, but $\mathrm{H}_{2} \mathrm{O}$ only in $\mathrm{T}$ Tauri sources. From this result we conclude that $\mathrm{H}_{2} \mathrm{O}$ far-IR emission is not detected in Herbig AeBe sources as a class and that the three sources with tentative detection through line stacking may be peculiar in this regard.

\section{5. $\mathrm{CH}^{+}$}

$\mathrm{CH}^{+}$emission is detected toward two Herbig Ae systems: HD 100546 and HD 97048 (Table 4). For HD 100546 six rotational lines are detected (see also Thi et al. 2011) while in the case of HD 97048 only the $J=6-5$ and $J=5-4$ transitions are seen. The line fluxes for HD 100546 differ from those reported by Thi et al. (2011) by $10-50 \%$ due to updated flux calibration (see Sect. 2.2).

\section{6. $[\mathrm{C} \mathrm{II}]$}

[C II] emission is detected toward 7 (out of 16) Herbig AeBe sources and 2 (out of 4) T Tauri stars (Table 3). In contrast with $[\mathrm{OI}]$, the [C II] emission is often spatially extended (e.g. Bruderer et al. 2012). This proves that some of the emission is produced in the large scale environment (cloud or remnant envelope) around the star even though very extended emission on $\gtrsim 6^{\prime}$ scales has been chopped out. More details are given in Appendix D where the [C II] spectral maps are also

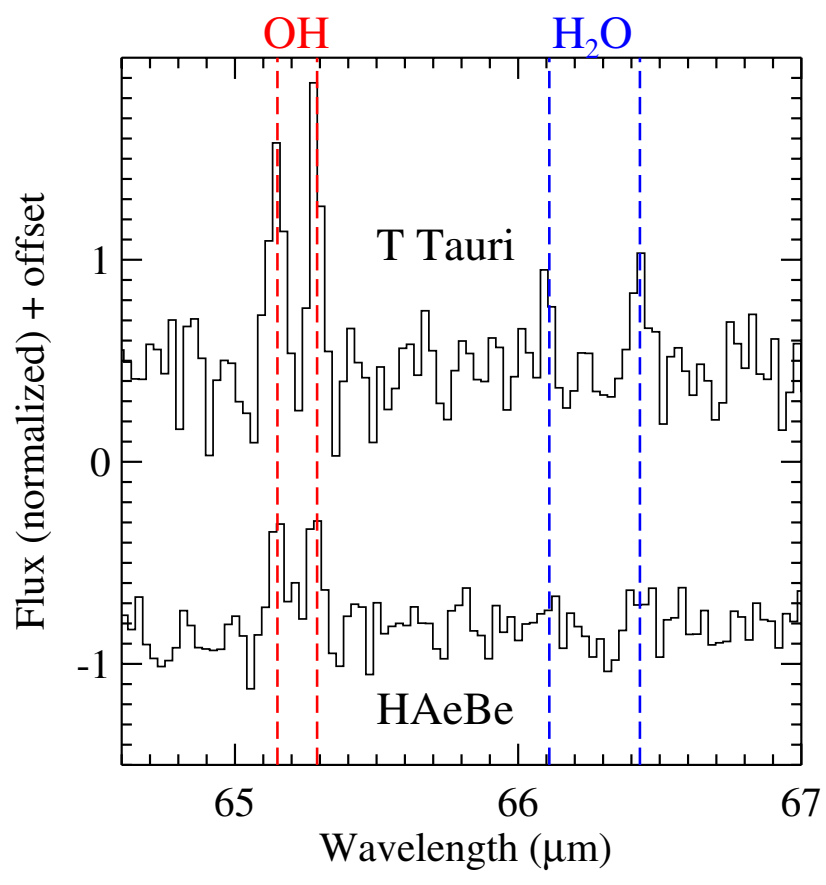

Fig. 2. Average PACS spectra for T Tauri and Herbig AeBe at $65 \mu \mathrm{m}$.

presented. The [C II] flux reported in Table 3 refers to the onsource spectrum only, that is the flux measured in the central spaxel after subtraction of the spatially extended emission (see Appendix D). These values must be considered an upper limit to the $[\mathrm{C} \mathrm{II}]$ emission arising from the disk as extended emission from a compact remnant envelope may still be present in the central 9.' $4 \times 9$ 9.' 4 area of the sky. The closest target is at $\sim 100 \mathrm{pc}$ and the size of the central spaxel corresponds to a physical scale of $\sim 1000 \mathrm{AU}$ which is of the same order as a compact envelope. Moreover, given the large PSF at this wavelength, some of the spatially extended emission will fall into the central spaxel.

Two of the sources presented here (AB Aur and HD 100546) have been previously observed at far-IR wavelengths with ISO-LWS (Giannini et al. 1999; Lorenzetti et al. 2002). The [OI] $63 \mu \mathrm{m}$ fluxes agree within $10-15 \%$, which is within the calibration uncertainty. For the [O I] $145 \mu \mathrm{m}$ line, the ISO flux is 1.5 times larger than the PACS value reported here. The [C II] fluxes are discrepant: in both cases, the flux measured with ISO is much larger (more than an order of magnitude) than the values reported here. This is due to the diffuse [C II] emission in the large (80") ISO beam which was not removed in the ISO observations.

\section{Analysis}

\subsection{Correlation of line luminosities}

The lines and continuum fluxes can show a correlation if the emitting conditions are physically linked. In particular, the emission of oxygen fine structure lines is expected to be correlated. We excluded the [C II] line from this analysis as the on-source flux (i.e. the flux emerging from the disk) is only an upper limit (see Sect. 3.6).

Figure 3 presents a series of plots of observed line luminosities versus each other and versus far-IR continuum. The plotted quantities are the logarithm of line luminosity $\left(\log \left(4 \pi d^{2} F_{\text {line }} / L_{\odot}\right)\right)$ and continuum luminosity at $63 \mu \mathrm{m}$ $\left(\log \left(4 \pi d^{2} F_{63 \mu \mathrm{m}} / L_{\odot}\right)\right)$. To search for possible correlations/trends, 

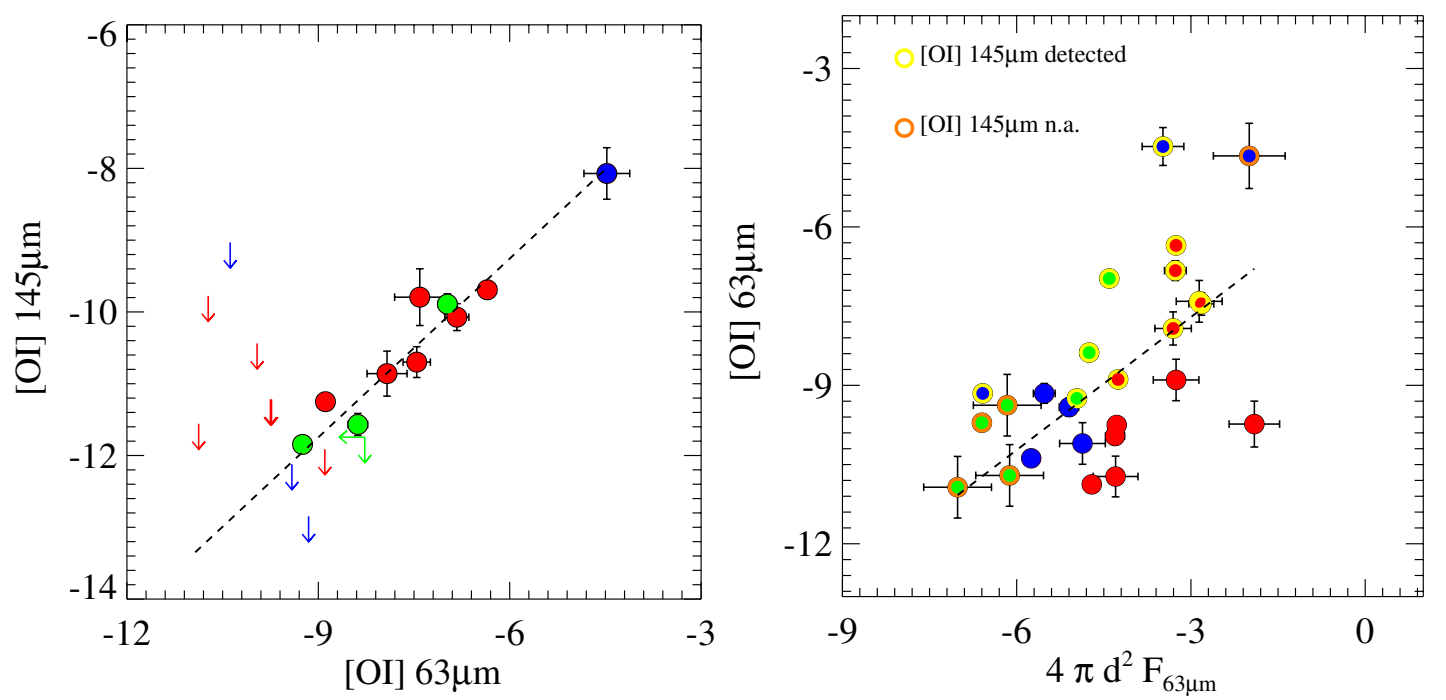

Fig. 3. Correlations plots. The left panel shows the correlation between the oxygen fine structure lines. In the right panel $F_{63 \mu \mathrm{m}}$ is the continuum flux at $63 \mu \mathrm{m}$ : open yellow circles indicate objects with [O I] $145 \mu \mathrm{m}$ detections; open orange circles indicate objects with [O I] $145 \mu \mathrm{m}$ data not available. Detections are plotted as filled circle and upper limits as arrows, red for HAeBe group I, blue for HAeBe group II, green for TTs. All luminosities are expressed in $L_{\odot}$ and are plotted on a logarithmic scale.

different statistical tests have been performed using the ASURV (Rev. 1.2 Isobe \& Feigelson 1990; Lavalley et al. 1992) statistical package which implements the methods presented in Isobe et al. (1986). In particular three different correlation tests have been used: Cox-Hazard regression, generalized $\tau$ Kendall, generalized $\rho$ Spearman. Linear regression coefficients are calculated with the EM algorithm. These statistical tests include upper limits.

As expected, a correlation is found between the [O I] $145 \mu \mathrm{m}$ and [O I] $63 \mu \mathrm{m}$ luminosities:

$\log L_{[\mathrm{O}]} 145 \mu \mathrm{m}=(0.83 \pm 0.06) \log L_{[\mathrm{O} \mathrm{I}]} 63 \mu \mathrm{m}-(4.28 \pm 0.47)$

The standard deviation is 0.28 . The three correlation tests give a probability that a correlation is not present of $<0.0002$.

We also searched for correlations between line and continuum flux. The only finding is that sources with stronger infrared continuum luminosity tend to have stronger [O I] $63 \mu \mathrm{m}$ line luminosity (Fig. 3, right panel)

$\log L_{[\mathrm{OI}] 63 \mu \mathrm{m}}=(0.84 \pm 0.20) \log L_{63 \mu \mathrm{m}}-(5.19 \pm 0.95)$

with a standard deviation of 1.45 . The three correlation tests give a probability of $<0.002$, suggesting that a correlation is indeed present. Nevertheless, the scatter is large: a high infrared continuum flux is a necessary but not sufficient condition to have stronger [O I] $63 \mu \mathrm{m}$ emission. No other clear correlations with source parameters are found. The origin of these correlations and the implications for the line emitting region are discussed in Sect. 5.2.

\section{2. [C $\mathrm{II}]-\left[\mathrm{O}_{\mathrm{I}}\right]$ diagnostic plot}

The atomic fine structure lines can be used as diagnostics of the physical conditions of the emitting gas. In this section we analyze the three line ratios: [O I] $145 \mu \mathrm{m} /[\mathrm{O} \mathrm{I}] 63 \mu \mathrm{m}$, [O I $145 \mu \mathrm{m} /[\mathrm{C} \mathrm{II}], \quad[\mathrm{O} \mathrm{I}] 63 \mu \mathrm{m} /[\mathrm{C}$ II $]$ The observed [OI] $63 \mu \mathrm{m} /[\mathrm{OI}] 145 \mu \mathrm{m}$ ratio goes from $10-40$ and it is higher than the typical ratio measured in molecular clouds (<10, e.g., Liseau et al. 1999). The gas density and the incident
FUV flux can be estimated by comparing the observations with PDR models.

In the high density regime $\left(n>10^{4} \mathrm{~cm}^{-3}\right)$ different PDR models do not agree and may predict very different gas temperatures (e.g. Röllig et al. 2007). Since the oxygen fine structure lines are very sensitive to the temperature, different models produce very different line ratios. The aim of our analysis is to look for a trend consistent with the observations. For this reason, the comparison of the data to a single PDR model is justified. The model used here is from Kaufman et al. (1999). With this choice we can directly compare our results with those of Lorenzetti et al. (2002) based on ISO data.

Figure 4 shows the observed line ratios and the model predictions. DG Tau was not included in this analysis as both the [OI] $63 \mu \mathrm{m}$ and [C II] lines are spatially extended and the onsource flux emission is an upper limit in both cases. According to this model, there is a group of sources (AB Aur, HD 50138, HD 97048, HD 100546, HD 179218) with gas density $n>$ $10^{5} \mathrm{~cm}^{-3}$ and $G_{0}$ between $10^{3}$ and $10^{6}$, where $G_{0}$ is the FUV $(6-13.6 \mathrm{eV})$ incident flux measured in units of the local galactic interstellar field ( $1 G_{0}=1.6 \times 10^{-3} \mathrm{erg} \mathrm{cm}^{-2} \mathrm{~s}^{-1}$, Habing 1968). These values correspond to surface temperatures $T_{\mathrm{S}} \sim 500 \mathrm{~K}-$ a few $10^{3} \mathrm{~K}$ at radii where most of the emission originates. The density is lower for IRS $48\left(\sim 10^{4} \mathrm{~cm}^{-3}\right)$ and HD 38120 (a few $10^{2} \mathrm{~cm}^{-3}$ ). As noted before, not all the [C II] emission measured with PACS comes from the same region as the oxygen lines, thus the intrinsic (disk) oxygen-carbon line ratio can be higher than what is found here. A lower $[\mathrm{CII}] /[\mathrm{OI}]$ ratio shifts the results to even higher gas density and temperature. For this reason the gas densities found in Fig. 4 should be considered as a lower limit to the gas density of the oxygen emitting region. The values of $n$ and $G_{0}$ found here are larger than those found with ISO for Herbig AeBe stars (Lorenzetti et al. 2002). The differences are driven by the higher [C II] flux measured with ISO (see Sect. 3.6). In general, the physical conditions derived here are consistent with disk surface layers.

\section{3. $\mathrm{OH}, \mathrm{H}_{2} \mathrm{O}$ and $\mathrm{CH}^{+}$excitation}

In this section the rotational diagrams of $\mathrm{OH}, \mathrm{H}_{2} \mathrm{O}$ and $\mathrm{CH}^{+}$ are analyzed. The measured Herschel/PACS line fluxes of all 
D. Fedele et al.: DIGIT survey of far-infrared lines from protoplanetary disks. I.

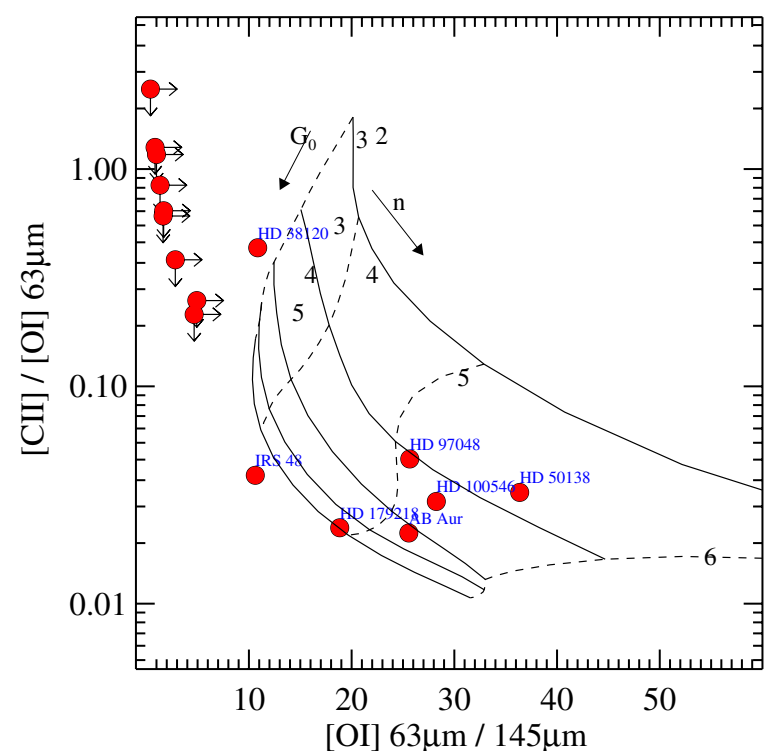

Fig. 4. Observed line ratios of the atomic fine structure lines and PDR model predictions. The arrows indicate the 3- $\sigma$ upper limits. The continuous lines indicate the region of constant $G_{0}$ for values $10^{2}-3.6 \times 10^{6}$. The dashed lines indicate the iso-density surface for values of $10^{2}-10^{6} \mathrm{~cm}^{-3}$.

sources are fit in a homogeneous way with a uniform slab of gas in local thermal equilibrium (LTE) including the effects of line opacity and line overlap (Bruderer et al. 2010). This is a simple model to provide estimates of the physical conditions in the regions where the lines arise. The gas column density derived here corresponds to the column density of a "warm" molecular layer.

\subsubsection{Slab model}

The molecular emission is assumed to emerge from a disk with homogeneous temperature and column density and a radius $r$. The solid angle is taken to be $\mathrm{d} \Omega_{\mathrm{s}}=\pi r^{2} / d^{2}$, where $d$ is the distance of the source. The flux of an optically thin line can be written as

$F_{\mathrm{ul}}=\mathrm{d} \Omega_{\mathrm{s}} \cdot I_{\mathrm{ul}}=\pi \frac{r^{2}}{d^{2}} \frac{h v_{\mathrm{ul}}}{4 \pi} A_{\mathrm{ul}} N_{\mathrm{mol}} \frac{g_{\mathrm{u}} \mathrm{e}^{-E_{\mathrm{u}} / k T}}{Q(T)}$

with the line frequency $v_{\mathrm{ul}}$, the Einstein-A coefficient $A_{\mathrm{ul}}$, the molecular column density $N_{\text {mol }}$, the statistical weight of the upper level $g_{\mathrm{u}}$, the energy of the upper level $E_{\mathrm{u}}$ and the partition function $Q(T)$. The molecular data are from the LAMDA database (Schöier et al. 2005). The number of emitting molecules is

$\mathcal{N}=\frac{4 \pi d^{2} F_{\mathrm{ul}} Q(T) \exp \left(E_{\mathrm{u}} / k T\right)}{h v_{\mathrm{ul}} A_{\mathrm{ul}} g_{\mathrm{u}}}$.

Rearranging Eq. (3) yields

$\mathrm{e}^{Y} \equiv \frac{4 \pi F_{\mathrm{ul}}}{A_{\mathrm{ul}} h v_{\mathrm{ul}} g_{\mathrm{u}}}=\pi \frac{r^{2}}{d^{2}} N_{\mathrm{mol}} \frac{\mathrm{e}^{-E_{\mathrm{u}} / k T}}{Q(T)} \equiv \pi \frac{r^{2}}{d^{2}} \frac{N_{\mathrm{u}}}{g_{\mathrm{u}}}$

Thus the vertical axis of a rotational diagram is given by

$Y=\ln \left(\frac{4 \pi F_{\mathrm{ul}}}{A_{\mathrm{ul}} h v_{\mathrm{ul}} g_{\mathrm{u}}}\right)=\ln \left(\pi \frac{r^{2}}{d^{2}} \frac{N_{\mathrm{mol}}}{Q(T)}\right)-\frac{E_{\mathrm{u}}}{k T}$

The free parameters of the model are the excitation temperature $T_{\text {ex }}$ and the column density $N_{\text {mol }}$. The emitting area can be determined uniquely for every given combination of $T_{\mathrm{ex}}$ and $N_{\mathrm{mol}}$.
Table 4. $\mathrm{CH}^{+}$line fluxes.

\begin{tabular}{lccc}
\hline \hline Transition & $\begin{array}{c}\text { Wavelength } \\
(\mu \mathrm{m})\end{array}$ & HD 100546 & HD 97048 \\
\hline$J=6-5$ & 60.25 & $18.5 \pm 2.0$ & $2.9 \pm 1.5$ \\
$J=5-4$ & 72.14 & $14.8 \pm 2.0$ & $2.2 \pm 0.5$ \\
$J=4-3$ & 90.02 & $13.1 \pm 2.0$ & $<3.0$ \\
$J=3-2$ & 119.86 & $3.6 \pm 1.5$ & $<2.5$ \\
$J=2-1$ & 179.60 & $4.2 \pm 1.5$ & $<2.7$ \\
\hline
\end{tabular}

Notes. Units and upper limits as in Table 3.

If all lines are optically thin, the column density and emitting area $\left(\pi r^{2}\right)$ are degenerate. In this case we can measure the total number of molecules $(\mathcal{N})$ and constrain the upper limit of $N_{\text {mol }}$ and the lower limit of $r$. For optically thick lines, the spectrum is calculated on a very fine wavelength grid using

$I_{v}=\mathrm{d} \Omega_{\mathrm{s}} B_{v}\left(T_{\mathrm{ex}}\right)\left(1-\mathrm{e}^{\tau_{v}}\right)$

with $\tau_{v}$ obtained from the sum of the

$\tau_{v}^{i}=\frac{A_{\mathrm{ul}} c^{2}}{8 \pi v^{2}}\left(N_{l} \frac{g_{\mathrm{u}}}{g_{l}}-N_{\mathrm{u}}\right) \phi_{v}$

over all fine structure components $(i=1,2, \ldots)$. Here, $\phi_{v}$ is the normalized line profile function, which is assumed to be a Gaussian with width corresponding to the thermal line width. No further (e.g. turbulent) line broadening is included. More details are given in Bruderer et al. (2010). For the analysis of the $\mathrm{H}_{2} \mathrm{O}$ lines an ortho-to-para ratio of 3 is assumed. The best fit parameters are found by minimizing the reduced $\chi^{2}\left(\tilde{\chi}^{2}\right)$ between model and observations.

\subsection{2. $\mathrm{OH}$}

$\mathrm{OH}$ rotational diagrams have been fitted only for sources for which 4 (or more) $\mathrm{OH}$ doublets have been detected. The $\mathrm{OH}$ rotational diagrams are presented in Figs. $5 \mathrm{a}$ and $5 \mathrm{~b}$ where the PACS measurements are shown as red dots and the best-fit model as blue stars. The figure also shows the $\tilde{\chi}^{2}$ contours of the fit to the data; that of HD 163296 is reported in Fedele et al. (2012). The blue contour represents the $1 \sigma$ confidence level of the fit which corresponds to $\tilde{\chi}^{2}=\operatorname{minimum}\left(\tilde{\chi}^{2}\right)+1$. The best fit results are reported in Table 6 . The $\mathrm{OH}$ emission is characterized by a warm temperature with $T_{\text {ex }} \sim 100-400 \mathrm{~K}$. In some cases all the $\mathrm{OH}$ lines are optically thin $\left(N_{\text {mol }} \lesssim 10^{15} \mathrm{~cm}^{-2}\right)$ and they fall on a straight line in the corresponding rotational diagram. For these sources, the $\mathrm{OH}$ column density and emitting radius are degenerate so only a lower boundary to the emitting radius is given, varying between 20 and $50 \mathrm{AU}$. The lowest excitation temperature is found for HD 50138 and DG Tau $\left(T_{\mathrm{ex}} \sim 100-130 \mathrm{~K}\right)$.

Given the large critical density of the far-IR $\mathrm{OH}$ lines and the strong infrared continuum, non-LTE excitation (including infrared pumping) can be important. We verified the effects of non-LTE excitation using RADEX (van der Tak et al. 2007). The detailed analysis is presented in Appendix C. The RADEX simulation shows that high gas densities $\left(n \geq 10^{10} \mathrm{~cm}^{-3}\right)$ are needed to reproduce the observed rotational diagram, even when a realistic infrared radiation field produced by the dust continuum is included in the RADEX simulation. The high density justifies the LTE assumption. 
Table 5. $\mathrm{H}_{2} \mathrm{O}$ line fluxes.

\begin{tabular}{ccc}
\hline \hline Transition & $\begin{array}{c}\text { Wavelength } \\
(\mu \mathrm{m})\end{array}$ & AS 205 \\
\hline $4_{32}-3_{21}$ & 58.71 & $2.5 \pm 1.1$ \\
$7_{26}-6_{15}$ & 59.99 & $2.4 \pm 0.7$ \\
$7_{16}-6_{25}$ & 66.09 & $3.0 \pm 1.1$ \\
$3_{30}-2_{21}$ & 66.44 & $2.9 \pm 1.1$ \\
$7_{07}-6_{16}$ & 71.96 & $3.1 \pm 1.1$ \\
$3_{21}-2_{12}$ & 75.39 & $2.0 \pm 0.8$ \\
$4_{23}-3_{12}$ & 78.74 & $2.7 \pm 1.0$ \\
$6_{15}-5_{24}$ & 78.93 & $2.8 \pm 1.0$ \\
$6_{06}-5_{15}$ & 83.29 & $1.7 \pm 0.7$ \\
$2_{21}-1_{10}$ & 108.07 & $1.0 \pm 0.4$ \\
$3_{03}-2_{12}$ & 174.62 & $<1.6$ \\
$2_{11}-1_{01}$ & 179.53 & $<1.6$ \\
\hline & & DG Tau \\
\hline $4_{23}-3_{12}$ & 78.75 & $1.2 \pm 0.6$ \\
$6_{16}-5_{05}$ & 82.03 & $1.3 \pm 0.7$ \\
$2_{21}-1_{10}$ & 108.13 & $1.3 \pm 0.4$ \\
$2_{12}-1_{01}$ & 179.54 & $1.1 \pm 0.4$ \\
\hline & & S CrA \\
\hline $8_{18}-7_{07}$ & 63.31 & $2.3 \pm 0.6$ \\
$7_{16}-6_{25}$ & 66.09 & $1.6 \pm 0.6$ \\
$7_{07}-6_{16}$ & 71.96 & $2.4 \pm 0.7$ \\
$4_{23}-3_{12}$ & 78.74 & $2.3 \pm 0.7$ \\
$4_{13}-4_{04}$ & 187.11 & $1.2 \pm 0.4$ \\
\hline
\end{tabular}

Notes. Units and upper limits as in Table 3.

\subsection{3. $\mathrm{H}_{2} \mathrm{O}$}

Figure 6 (right) shows the $\tilde{\chi}^{2}$ contours for AS 205, best fit results are given in Table 6. The molecular data are listed in Table B.2. The best fit models (first $\tilde{\chi}^{2}$ contour) give $T_{\mathrm{ex}} \sim 100-300 \mathrm{~K}$, a column density $N_{\text {mol }}>10^{17} \mathrm{~cm}^{-2}$ and an emitting radius $r \sim 10-30$ AU. The rotational diagram is shown in Fig. 6 (left) together with the model predictions. The model reproduces well the measured line fluxes $\left(\tilde{\chi}^{2}=0.5\right)$ as well as the upper limits of the low-energy backbone lines, $3_{03}-2_{12}\left(E_{\mathrm{u}}=196 \mathrm{~K}\right)$ and $2_{12}-1_{01}\left(E_{\mathrm{u}}\right)=114 \mathrm{~K}$. According to the slab model, the detected lines are optically thick with optical depth $\tau \sim 1-10$, so the inferred number of molecules is a lower limit. In the case of LTE, the line flux ratio of the low and high energy lines decreases quickly with increasing temperature. To test the validity of the LTE assumption, we checked the line flux ratio of a lowenergy transition $2_{12}-1_{01}(179 \mu \mathrm{m}$ non detected) versus a highenergy one $7_{07}-6_{16}(72 \mu \mathrm{m})$. The observed ratio is $<0.5(3 \sigma$ upper limit). According to the LTE model, this ratio drops below 0.5 for $T>65 \mathrm{~K}$ and $N>10^{17} \mathrm{~cm}^{-2}$. Low gas temperatures $(T<65 \mathrm{~K})$ are ruled out by the detection of high-energy transitions. Large column densities $\left(N_{\mathrm{H}_{2} \mathrm{O}}>10^{17} \mathrm{~cm}^{-2}\right)$ are needed to reproduce the observed scatter (deviation from optically thin) in the rotational diagram.

Non-LTE excitation may also be important because of the large critical densities of the $\mathrm{H}_{2} \mathrm{O}$ lines detected here $\left(n_{\text {crit }}=\mathrm{a}\right.$ few $10^{9} \mathrm{~cm}^{-3}$ for $T=300 \mathrm{~K}$ ), and the scatter of the $\mathrm{H}_{2} \mathrm{O}$ lines in the rotational diagram can also be produced by sub-thermal excitation. If this is the case, the kinetic temperature of the $\mathrm{H}_{2} \mathrm{O}$ containing gas is likely larger than the excitation temperature (e.g., Herczeg et al. 2012). However, given the results of the $\mathrm{OH}$ modeling, the far-IR $\mathrm{H}_{2} \mathrm{O}$ emission likely comes from a high gas density region $\left(n \geq 10^{10} \mathrm{~cm}^{-3}\right)$ where the $\mathrm{H}_{2} \mathrm{O}$ rotational levels are in LTE.
Table 6. Best fit results of the slab model.

\begin{tabular}{lcccc}
\hline \hline & $\begin{array}{c}T_{\text {ex }} \\
{[\mathrm{K}]}\end{array}$ & $\begin{array}{c}N_{\text {mol }} \\
{\left[\mathrm{cm}^{-2}\right]}\end{array}$ & $\begin{array}{c}r \\
{[\mathrm{AU}]}\end{array}$ & $\log (\mathcal{N})$ \\
\hline \multicolumn{5}{c}{$\mathrm{OH}$} \\
\hline AS 205 & 190 & $8 \times 10^{15}$ & 19 & 45.31 \\
DG Tau & 115 & $4 \times 10^{15}$ & 50 & 45.85 \\
AB Aur & 190 & $<10^{14}$ & $>50$ & 44.25 \\
HD 36112 & 240 & $<10^{14}$ & $>50$ & 44.25 \\
HD 50138 & 130 & $2 \times 10^{15}$ & 95 & 46.10 \\
HD 100546 & 210 & $2 \times 10^{14}$ & 40 & 44.35 \\
HD 104237 & 160 & $2 \times 10^{15}$ & 20 & 44.75 \\
HD 163296 & 425 & $8 \times 10^{14}$ & 15 & 44.10 \\
\hline \multicolumn{5}{c}{$\mathrm{H}_{2} \mathrm{O}$} \\
\hline AS 205 & $100-300$ & $>10^{17}$ & $10-30$ & $>45.85$ \\
HD 163296 & $250-300$ & $10^{14}-10^{15}$ & 20 & $43.5-44.5$ \\
\hline \multicolumn{5}{c}{$\mathrm{CH}^{+}$} \\
\hline HD 100546 & $80-120$ & $10^{16}-10^{17}$ & $50-70$ & 46.94 \\
\hline
\end{tabular}

Notes. Upper and lower limits are bounds from the modeling.

In the case of DG Tau, S CrA and RNO 90 only a few $\mathrm{H}_{2} \mathrm{O}$ lines are detected and the fit is not constrained.

The analysis of HD 163296 is reported in Fedele et al. (2012), who find that the far-IR $\mathrm{H}_{2} \mathrm{O}$ emission is optically thin, $N_{\text {mol }} \lesssim 10^{15} \mathrm{~cm}^{-2}$ with an emitting radius $r \sim 20 \mathrm{AU}$, and the excitation temperature is $T_{\mathrm{ex}} \sim 200-300 \mathrm{~K}$ (Table 6 ). For the other Herbig AeBe sources, HD 142527 and HD 104237, the individual $\mathrm{H}_{2} \mathrm{O}$ lines are too weak (below $3 \sigma$ ) for such an analysis. With the assumption of optically thin emission, the upper limit to the number of warm water molecules is given by Eq. (4). The $707-6_{16}$ line at $72 \mu \mathrm{m}$ is used. The partition sum is taken from the HITRAN TIPS program (Laraia et al. 2011) The typical upper limit to the total number of warm water molecules ranges $\sim$ a few $10^{43}-10^{45}$ for $T=300 \mathrm{~K}$. This number decreases by $\sim 20 \%$ for a gas temperature $T=400 \mathrm{~K}$. Assuming a characteristic emitting radius of $30 \mathrm{AU}$ the upper limit to the water column density, $N_{\text {mol }}=\mathcal{N}\left(\mathrm{H}_{2} \mathrm{O}\right) /\left(\pi r^{2}\right)$, is a few $10^{14} \mathrm{~cm}^{-2}\left(10^{15} \mathrm{~cm}^{-2}\right.$ for HD 50138).

\subsection{4. $\mathrm{CH}^{+}$}

The $\mathrm{CH}^{+}$rotational diagram for HD 100546 is shown in Fig. 7. The model that best fits the data gives $T_{\mathrm{ex}} \sim 80-120 \mathrm{~K}$, $N_{\text {mol }} \sim 10^{16}-10^{17} \mathrm{~cm}^{-2}$ and $r \sim 50-70 \mathrm{AU}$. The column density is not well constrained because of the large uncertainty in the two lower $J$ lines.

Compared to Thi et al. (2011), the slab model analysis presented here indicates a lower excitation temperature, $T_{\mathrm{ex}} \sim 100 \mathrm{~K}$ versus $323_{-151}^{+2320} \mathrm{~K}$ (Thi et al. 2011). The $\mathrm{CH}^{+}$emitting region is also different: Thi et al. (2011) find that most of the emission comes from a narrow rim at the cavity edge between 10-13 AU from the star through full thermo-chemical modeling, whereas the slab model suggests a much larger emitting area. Part of the discrepancy is due to a different flux calibration of the PACS spectra; the PACS spectrum presented here matches the PACS photometric points which have an accuracy of $5 \%$ in absolute flux. 
D. Fedele et al.: DIGIT survey of far-infrared lines from protoplanetary disks. I.

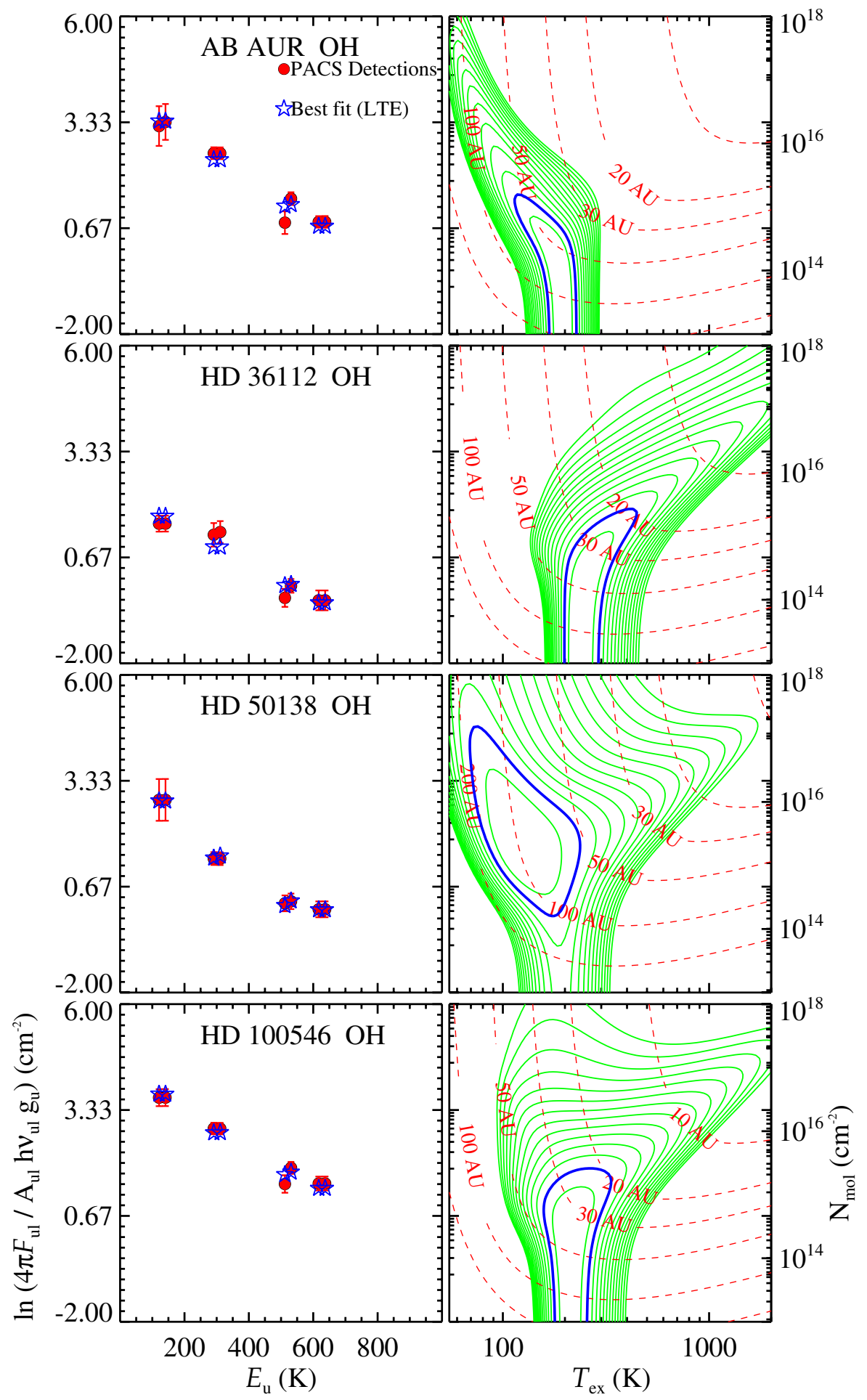

Fig. 5. (Left) $\mathrm{OH}$ rotational diagram. PACS detections are plotted as (red) dots, best-fit model is shown as (blue) stars. (Right) $\tilde{\chi}^{2}$ contours. The $1 \sigma$ confidence level is highlighted by a (blue) thick line. The (red) dashed lines represent the emitting radius. The green lines are the $\tilde{\chi}^{2}$ contours in steps of 0.5 .

\section{Discussion}

\subsection{Origin of far-IR emission lines}

According to the results of the $\mathrm{OH}$ rotational diagram, the far-IR $\mathrm{OH}$ lines are emitted by warm gas with a $T_{\text {ex }}$ of $100-400 \mathrm{~K}$. The $\mathrm{OH}$ emission toward AB Aur, HD 36112, HD 100546 is optically thin $\left(N<10^{15} \mathrm{~cm}^{-2}\right)$. For the remaining sources (AS 205, DG Tau, HD 50138, HD 163296) the far-IR OH lines are at the border between optically thick and optically thin emission
$\left(N \sim 10^{15}-10^{16}\right)$. The derived excitation temperature and the emitting radius are consistent with a disk origin with the emission coming from the upper layers of the disk at distances of 15$50 \mathrm{AU}$ from the star (100 AU in the case of HD 50138). Given the high excitation temperature and high critical densities (on the order of $10^{8} \mathrm{~cm}^{-3}$ to excite $\mathrm{OH}$ ), the emitting radius cannot be much larger.

In the case of the T Tauri system DG Tau, the derived excitation temperature is $115 \mathrm{~K}$ (lowest in the sample) and the 


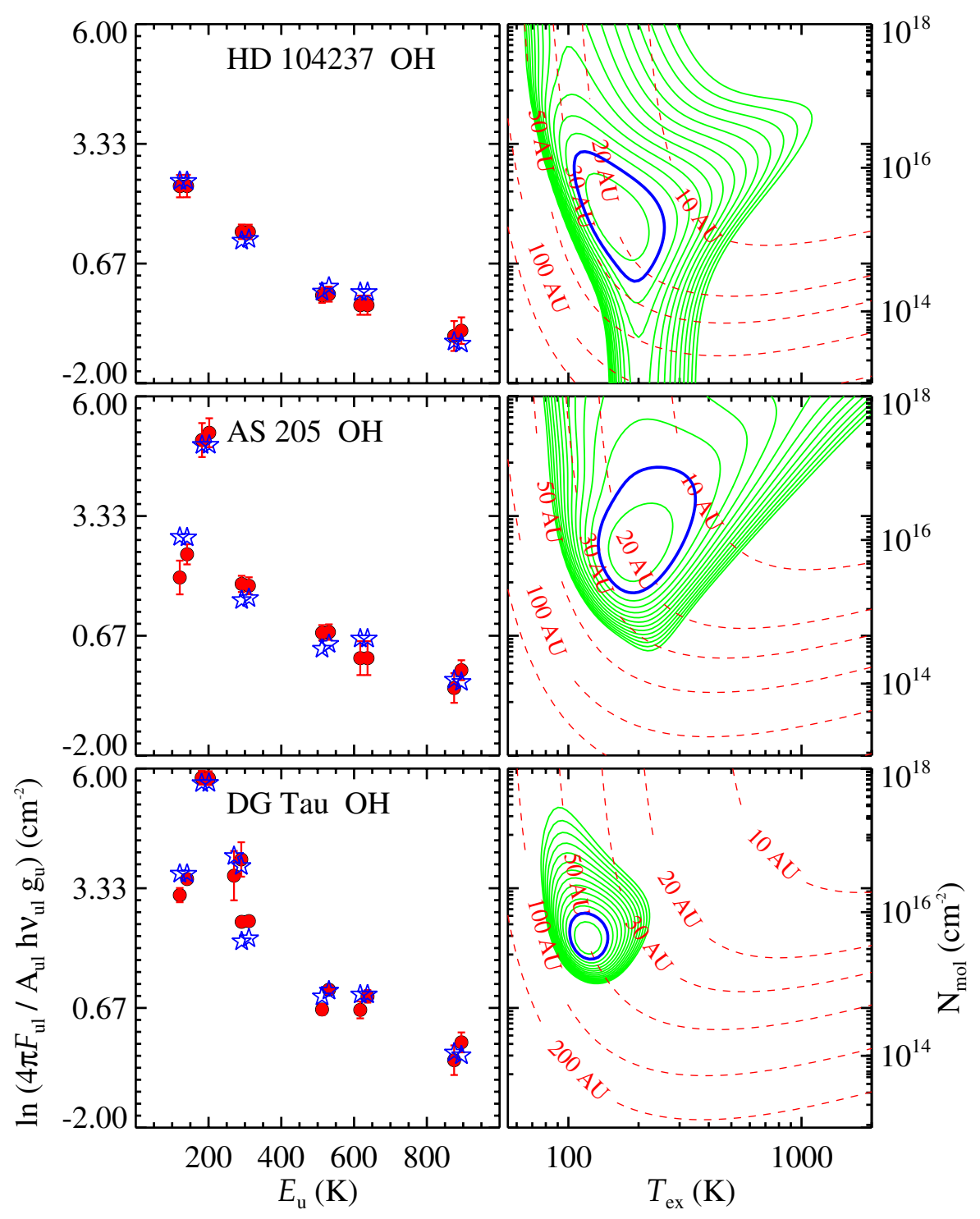

Fig. 5. continued.

$\mathrm{OH}$ emitting radius is $\sim 50 \mathrm{AU}$, which is 2.5 times larger than the emitting radius of the other T Tauri star (AS 205). In this case, a further contribution to the $\mathrm{OH}$ lines may come from a shock associated with the molecular outflow/jet associated with the system. Indeed, compact warm $\mathrm{OH}$ emission has been observed to be associated with outflows in embedded young stellar objects (van Kempen et al. 2010; Wampfler et al. 2010, 2013; Podio et al. 2012; Karska et al. 2013). Further analysis including the mid-infrared lines (from Spitzer) is needed to disentangle the disk/outflow origin of $\mathrm{OH}$ for these systems. In the case of AS 205 the emitting redius of the far-IR $\mathrm{H}_{2} \mathrm{O}$ lines is $\sim 10-30 \mathrm{AU}$, also consistent with a disk origin.

As discussed in Sect. 4.2, the high density and UV fluxes implied by the ratios of the atomic fine structure lines are consistent with a disk origin for most of the sources. The [C II] emission is spatially extended in all sources where the line is detected. This suggests that there is a contribution from a diffuse cloud (or remnant envelope) around the young star. The on-source [C II] flux correlates with the oxygen line fluxes suggesting that (some of) the on-source [C II] emission is associated with the disk. From the PACS spectra it is however impossible to disentangle the disk emission from the diffuse emission. HIFI spectra of the [C II] line profiles are needed to solve this issue (Fedele et al. 2013).

\subsection{Disk geometry and dust settling}

The protoplanetary disks presented here vary in geometry and degree of grain growth and settling. These factors are important for the excitation of the atomic and molecular gas. For example, in the case of water, a combination of these factors can play a role in the low detection rate toward Herbig AeBe systems. As pointed out by Woitke et al. (2009), whether or not the puffed-up inner rim shadows the hot water layer is important, since shadowing reduces the UV radiation field by about two orders of magnitude and increases water by the same amount. Also, grain settling, presence or absence of PAHs and the gas-to-dust ratio can all have a large effect in boosting line fluxes (e.g., Meijerink et al. 2009; Najita et al. 2011; Tilling et al. 2012; Bruderer et al. 2012).

Far-IR CO emission is only detected in HAeBe systems of Group I (Table 2 and Paper II). The high- $J$ CO lines detected with PACS are sensitive to the UV flux impinging onto the 

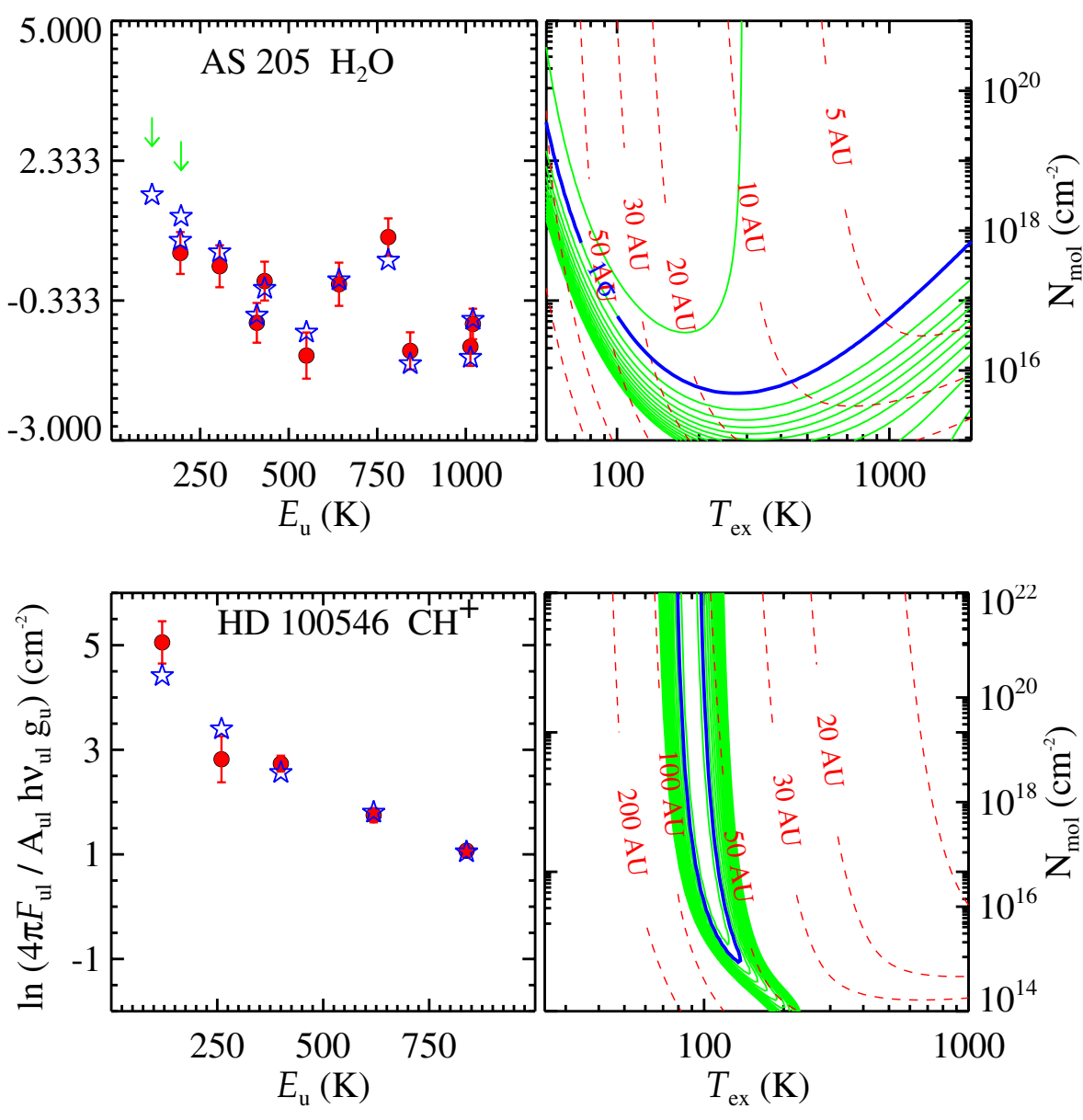

Fig. 6. $\mathrm{H}_{2} \mathrm{O}$ rotational diagram $($ Left $)$ and $\tilde{\chi}^{2}$ contours (right) for AS 205. Colors and symbols as in Fig. 5. The (green) arrows indicate the $3 \sigma$ upper limits.
Fig. 7. $\mathrm{CH}^{+}$rotational diagram (left) and $\tilde{\chi}^{2}$ contours (right) for HD 100546. Colors and symbols as in Fig. 5. disk, which controls the disk gas temperature. Using the thermochemical models of Bruderer et al. (2012), we have shown that flared disks indeed have higher gas temperatures out to several tens of $\mathrm{AU}$ and stronger high- $J \mathrm{CO}$ fluxes (Paper II). This is an independent proof that the disks of Group I indeed have a flared geometry. On the other hand, the $\mathrm{OH}$ lines are less sensitive to the gas temperature, consistent with its detection in both Group I and II sources (Bruderer et al., in prep., and Sect. 5.4).

The [OI] $63 \mu \mathrm{m}$ line is detected in most of the disks independently from disk geometry and stellar parameters. The high detection rate toward Group II sources is interesting. If these sources are indeed self-shadowed and/or have grain growth and settling, the gas temperature in the surface layers should be lower (Jonkheid et al. 2007) and the atomic and molecular emission at far-IR wavelengths is expected to be reduced in Group II disks. The excitation of the $\mathrm{O}\left({ }^{3} \mathrm{P}_{1}\right)$ level (upper level of the [O I] $63 \mu \mathrm{m}$ line) is mostly due to collisions with $\mathrm{H}$ and $\mathrm{H}_{2}$. Once the gas density exceeds the critical density of the line ${ }^{2}$ the excitation depends only on the temperature and no longer on the density. In this scenario, Group I sources can have stronger [O I] $63 \mu \mathrm{m}$ and [O I] $145 \mu \mathrm{m}$ emission due to the higher temperature of the gas. The intensity of the [OI] $63 \mu \mathrm{m}$ line varies by two orders of magnitude for a given value of the continuum flux at $63 \mu \mathrm{m}$. This implies a different gas density structure (in the [O I] forming region) from object to object. According to model predictions (e.g. Woitke et al. 2009; Bruderer et al. 2012), the oxygen emitting region can be more extended than the far-IR continuum. The far-IR continuum emission comes mostly from the inner

\footnotetext{
$2 n_{\text {crit }}([\mathrm{OI}] 63 \mu \mathrm{m})=2.5 \times 10^{5} \mathrm{~cm}^{-3}$ at $100 \mathrm{~K}$ and lower for higher temperatures.
}

$\sim 50 \mathrm{AU}$, while the oxygen lines originate in the outer disk (up to a few $10^{2} \mathrm{AU}$ in the case of Herbig AeBe stars). The large spread in [O I] $63 \mu \mathrm{m}$ fluxes for a given far-IR continuum flux (Fig. 3) suggests that the [OI]-bright sources may have an enhanced scale-height (more flared) compared to the [O I]-faint sources. Based on the results of Sect. 4.2 and on the high detection rate of [O I] $63 \mu \mathrm{m}$ we conclude that the oxygen lines have a disk origin in most of the cases, with the strength determined by the specific disk structure.

\subsection{Comparison to near- and mid-IR spectroscopy}

The comparison of the far-IR spectra shown here to the near$(1-5 \mu \mathrm{m})$ and mid- $(10-40 \mu \mathrm{m})$ IR spectra can give insights on the radial distribution of different gas species in the upper layers of protoplanetary disks. At longer wavelengths also larger vertical depths into the disk are probed.

Figure 8 shows the $\mathrm{OH} / \mathrm{H}_{2} \mathrm{O}$ column density ratio for T Tauri and Herbig AeBe stars measured at different wavelengths. The values represent the ratio of the total number of molecules and are taken from Salyk et al. (2008), Fedele et al. (2011), Salyk et al. (2011a) and from this work. In the case of Herbig AeBe stars, the mid-IR ratio refers to the ratio of the upper limits and is thus not constrained. At all wavelength ranges, the Herbig AeBe disks show a higher $\mathrm{OH} / \mathrm{H}_{2} \mathrm{O}$ abundance ratio compared to $\mathrm{T}$ Tauri disks. For Herbig AeBe systems the $\mathrm{OH} / \mathrm{H}_{2} \mathrm{O}$ lower limit does not vary much from near- to far-IR wavelengths. In contrast the $\mathrm{T}$ Tauri disks do show a clear decrease in the $\mathrm{OH} / \mathrm{H}_{2} \mathrm{O}$ ratio. The difference between the mid- and far-IR ratios are not significant. 


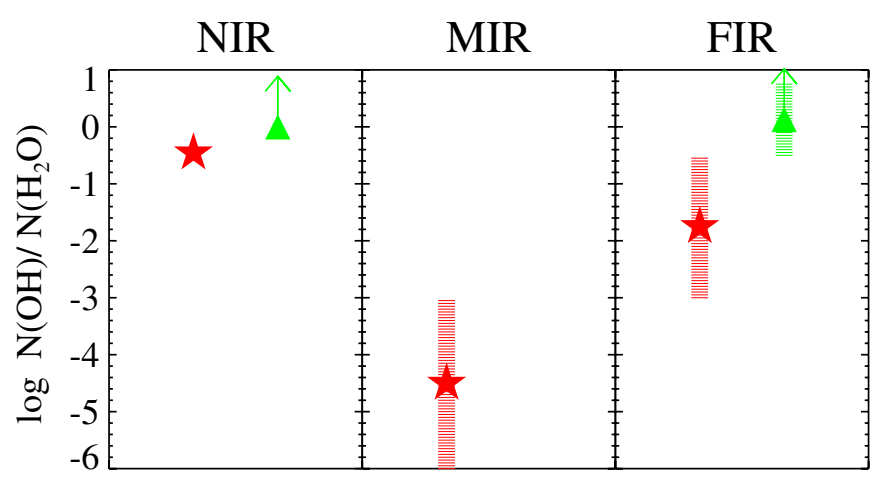

Fig. 8. $\mathrm{OH} / \mathrm{H}_{2} \mathrm{O}$ column density ratio for $\mathrm{T}$ Tauri (red stars) and Herbig AeBe (green squares) sources. The dashed regions indicate the range of the observed ratio.

Herbig AeBe: the major difference between short and long wavelengths is the lack of any $\mathrm{H}_{2} \mathrm{O}$ lines at near-IR (Mandell et al. 2008; Fedele et al. 2011) whereas weak $\mathrm{H}_{2} \mathrm{O}$ lines are detected at mid- and far-IR in some sources (Pontoppidan et al. 2010; Fedele et al. 2012; Meeus et al. 2012). Even though the detection rate of warm $\mathrm{H}_{2} \mathrm{O}$ is low and the individual lines are weak $(<\mathrm{a}$ few $10^{-17} \mathrm{~W} \mathrm{~m}^{-2}$ ), this finding suggests a different $\mathrm{H}_{2} \mathrm{O}$ abundance between the inner and outer disk. However, as Fig. 8 shows, the $\mathrm{OH} / \mathrm{H}_{2} \mathrm{O}$ abundance limits are similar at all wavelengths. Also interesting is the high detection rate $(\sim 40 \%)$ of far-IR OH emission for sources with full spectral coverage. This emission is detected in both Group I and II sources in contrast to near-IR OH emission which is detected in Group I sources only (Fedele et al. 2011). Finally, near-IR CO emission (ro-vibrational) is detected in several Herbig AeBe systems independently from disk geometry in contrast to far-IR CO which is only detected in flared disks (Paper II). This difference is likely a consequence of the larger number of UV photons that impinge onto the disk surface in the case of a flared geometry which can heat the gas to the larger distances responsible for the far-IR lines.

$T$ Tauri: the PACS spectra presented here are rich in molecular emission similar to the near-IR and mid-IR spectra. AS 205 and $\mathrm{RNO} 90$ have a rich $\mathrm{OH}$ and $\mathrm{H}_{2} \mathrm{O}$ spectrum ranging from the pure-rotational lines detected here to ro-vibrational lines detected with NIRSPEC and CRIRES $(3 \mu \mathrm{m})$ and Spitzer/IRS $(10-40 \mu \mathrm{m})$ (Salyk et al. 2011b; Pontoppidan et al. 2010; Mandell et al. 2012). The energy levels involved range from a few hundred to a few thousand $\mathrm{K}$. The $\mathrm{H}_{2} \mathrm{O}$-rich PACS spectrum is unlikely to originate from the same region of the disk as the shorter wavelength data. Indeed, based on the slab model, the emitting region of the far-IR lines of $\mathrm{OH}$ and $\mathrm{H}_{2} \mathrm{O}$ has a radius $r \sim 20-30 \mathrm{AU}$ in contrast to a radius of a few AU (or less) for the hot $\mathrm{H}_{2} \mathrm{O}$ near-IR and mid-IR lines (Salyk et al. 2011b). The conclusion is that $\mathrm{H}_{2} \mathrm{O}$ is present in the surface layers of disks around low-mass pre-main sequence stars from the inner ( $\sim 0.1 \mathrm{AU})$ out to the outer ( $\sim 30 \mathrm{AU})$ disk, but the $\mathrm{OH} / \mathrm{H}_{2} \mathrm{O}$ abundance ratio changes with radius (see Zhang et al. 2013, for the analysis of $\mathrm{H}_{2} \mathrm{O}$ data of one source).

\subsection{Disk chemistry and molecular excitation}

The different detection rates and excitation mechanisms of the various species provide information about the chemical processes governing the atmosphere of protoplanetary disks. In the case of the UV-bright Herbig stars, the chemistry and excitation are regulated more strongly by photoprocesses like UV fluorescence (e.g. for $\mathrm{CO}$ ) and photodissociation of molecules (e.g. $\mathrm{OH}, \mathrm{H}_{2} \mathrm{O}$ ) than in T Tauri stars, unless those low-mass sources have significant UV excess due to accretion.

First, we find that $\mathrm{OH}$ far-IR emission is detected in all groups of sources in contrast to $\mathrm{CO}$ far-IR emission which is only detected in flared Herbig AeBe disks and T Tauri disks. The excitation of the high- $J$ CO rotational lines in disks is regulated by the gas temperature which in turn is controlled by the $\mathrm{UV}$ radiation field. In the case of $\mathrm{OH}$, the lines are excited either by collisions with atomic and molecular hydrogen or through infrared pumping, both of which are much less sensitive to geometry. A third viable mechanism for $\mathrm{OH}$ is the prompt emission after the $\mathrm{OH}$ molecules are produced rotationally excited by the photodissociation of water which is also controlled by the UV field.

Another interesting finding is the detection of (weak) farIR $\mathrm{H}_{2} \mathrm{O}$ emission. The non-detection of hot $\mathrm{H}_{2} \mathrm{O}$ lines at nearand mid-IR wavelengths suggests that the atmospheres of disks around early type stars lack $\mathrm{H}_{2} \mathrm{O}$ molecules due the photodissociation of $\mathrm{H}_{2} \mathrm{O}$ by the strong UV radiation field of the central star (Fedele et al. 2011; Pontoppidan et al. 2010). In contrast, the PACS detection of warm $\mathrm{H}_{2} \mathrm{O}$ in some sources suggests that $\mathrm{H}_{2} \mathrm{O}$ molecules can survive at large distances ( $>30 \mathrm{AU}$ ) from the star and somewhat deeper into the disk, likely produced by high temperature reactions of $\mathrm{O}+\mathrm{H}_{2}$ and $\mathrm{OH}+\mathrm{H}_{2}$ driving much of the oxygen into water (e.g., Bergin 2011; Woitke et al. 2010). The importance of this result is that it reveals the presence of an $\mathrm{H}_{2} \mathrm{O}$ reservoir in the outer disk around early type stars, beyond the traditional snow line.

$\mathrm{CH}^{+}$emission is found toward HD 100546 and HD 97048. Interestingly these are the only two Herbig AeBe sources where ro-vibrational $\mathrm{H}_{2}$ emission has been detected so far (Carmona et al. 2011). The velocity profile of the line suggests extended $\mathrm{H}_{2}$ emission to more that $50 \mathrm{AU}$ (radius) from the star (Carmona et al. 2011). This is likely the same spatial region traced by the far-IR $\mathrm{CH}^{+}$lines reported here: the $\mathrm{CH}^{+}$emitting area in HD 100546 is 50-70 AU according to the slab model. The detection of vibrationally excited $\mathrm{H}_{2}$ and $\mathrm{CH}^{+}$toward the same sources is not a coincidence but relates to the gas phase reaction (Sternberg \& Dalgarno 1995)

$\mathrm{C}^{+}+\mathrm{H}_{2}^{*} \rightarrow \mathrm{CH}^{+}+\mathrm{H}$.

If $\mathrm{H}_{2}^{*}$ is vibrationally excited, the forward reaction (which is endothermic by $\sim 4000 \mathrm{~K}$ ) is faster (see also Agúndez et al. 2010; Thi et al. 2011).

\section{Conclusion}

We present far-IR spectra of Herbig AeBe and T Tauri stars taken with Herschel/PACS. Besides the fine structure lines of [O I] and [C II], emission is detected of $\mathrm{CO}$ (Paper II), OH, $\mathrm{H}_{2} \mathrm{O}$ and $\mathrm{CH}^{+}$. The most common feature detected is the [O I] $63 \mu \mathrm{m}$ line.

Far-IR OH emission is detected in several sources. An LTE slab model including optical depth effects is used to fit the $\mathrm{OH}$ rotational diagram. The $\mathrm{OH}$ lines are likely associated with the disk, probing a warm layer of gas in the outer disk ( $r \gtrsim 20 \mathrm{AU})$. In contrast to the high- $J$ CO lines, the $\mathrm{OH}$ lines are detected in both flat and flared disks (Group I and II) around Herbig AeBe stars. The reason for this different may be the different excitation mechanisms for the two species.

Warm $\mathrm{H}_{2} \mathrm{O}$ emission is detected in three Herbig AeBe sources and in four $\mathrm{T}$ Tauri sources. In the Herbig sources, the emission is weak and the detection of warm $\mathrm{H}_{2} \mathrm{O}$ is confirmed 
D. Fedele et al.: DIGIT survey of far-infrared lines from protoplanetary disks. I.

only by line stacking. This result reveals the presence of an $\mathrm{H}_{2} \mathrm{O}$ reservoir in the outer disk region around Herbig stars. However, the $\mathrm{OH} / \mathrm{H}_{2} \mathrm{O}$ abundance limit is the same between inner and outer disk, consistent with a decreasing UV field. In the case of the T Tauri star AS 205 the slab model suggests "warm" $\left(T_{\mathrm{ex}} \sim\right.$ $100-300 \mathrm{~K}) \mathrm{H}_{2} \mathrm{O}$ emission coming from the inner 10-30 AU from the star. Overall, the $\mathrm{OH} / \mathrm{H}_{2} \mathrm{O}$ column density ratio decreases from inner and outer disk for $\mathrm{T}$ Tauri disks.

The flux ratios of atomic fine structure lines are fitted with PDR models involving high gas density $\left(n>10^{5} \mathrm{~cm}^{-3}\right)$ and high UV radiation field $\left(G_{\mathrm{o}} \sim 10^{3}-10^{7}\right)$ as expected for the atmosphere of protoplanetary disks. The presence of spatially extended [C II] emission (on scale of $10^{3} \mathrm{AU}$ ) implies the presence of diffuse material (e.g., remnant of the molecular cloud) around the young stars.

Acknowledgements. Support for this work, part of the Herschel open time key project program, was provided by NASA through an award issued by the Jet Propulsion Laboratory, California Institute of Technology. We are grateful to the DIGIT team for stimulating discussions and scientific support. Astrochemistry in Leiden is supported by the Netherlands Research School for Astronomy (NOVA), by a Spinoza grant and grant 614.001.008 from the Netherlands Organisation for Scientific Research (NWO), and by the European Community's Seventh Framework Programme FP7/2007-2013 under grant 238258 (LASSIE) and grant 291141 (CHEMPLAN). PACS has been developed by a consortium of institutes led by MPE (Germany) and including UVIE (Austria); KU Leuven, CSL, IMEC (Belgium); CEA, LAM (France); MPIA (Germany); INAF-IFSI/OAA/OAP/OAT, LENS, SISSA (Italy); IAC (Spain). This development has been supported by the funding agencies BMVIT (Austria), ESAPRODEX (Belgium), CEA/CNES (France), DLR (Germany), ASI/INAF (Italy), and CICYT/MCYT (Spain). The research of M.G. has been supported by the Austrian Research Promotion Agency (FFG) through the ASAP initiative of the Austrian Federal Ministry for Transport, Innovation and Technology (BMVIT). We thank the anonymous referee for the helpful comments.

\section{References}

Acke, B., \& van den Ancker, M. E. 2004, A\&A, 426, 151

Acke, B., Min, M., van den Ancker, M. E., et al. 2009, A\&A, 502, L17

Agúndez, M., Goicoechea, J. R., Cernicharo, J., Faure, A., \& Roueff, E. 2010, ApJ, 713, 662

Bast, J. E., Brown, J. M., Herczeg, G. J., van Dishoeck, E. F., \& Pontoppidan, K. M. 2011, A\&A, 527, A119

Bergin, E. A. 2011, in Physical process in circumstellar disks around young stars, ed. P. J. V. Garcia (Chicago: University of Chicago Press), 55

Bergin, E. A., Cleeves, L. I., Gorti, U., et al. 2013, Nature, 493, 644

Blake, G. A., \& Boogert, A. C. A. 2004, ApJ, 606, L73

Brittain, S. D., Rettig, T. W., Simon, T., et al. 2003, ApJ, 588, 535

Bruderer, S., Benz, A. O., Stäuber, P., \& Doty, S. D. 2010, ApJ, 720, 1432

Bruderer, S., van Dishoeck, E. F., Doty, S. D., \& Herczeg, G. J. 2012, A\&A, 541, A91

Carmona, A., van der Plas, G., van den Ancker, M. E., et al. 2011, A\&A, 533, A39

Carr, J. S., \& Najita, J. R. 2008, Science, 319, 1504

Carr, J. S., \& Najita, J. R. 2011, ApJ, 733, 102

Carr, J. S., Tokunaga, A. T., \& Najita, J. 2004, ApJ, 603, 213

Creech-Eakman, M. J., Chiang, E. I., Joung, R. M. K., Blake, G. A., \& van Dishoeck, E. F. 2002, A\&A, 385, 546

Doppmann, G. W., Najita, J. R., Carr, J. S., \& Graham, J. R. 2011, ApJ, 738, 112

Fedele, D., van den Ancker, M. E., Acke, B., et al. 2008, A\&A, 491, 809

Fedele, D., Pascucci, I., Brittain, S., et al. 2011, ApJ, 732, 106

Fedele, D., Bruderer, S., van Dishoeck, E. F., et al. 2012, A\&A, 544, L9

Fedele, D., Bruderer, S., van Dishoeck, E. F., et al. 2013, ApJ, 776, L3

Giannini, T., Lorenzetti, D., Tommasi, E., et al. 1999, A\&A, 346, 617

Green, J. D., Evans, N. J. II, Jørgensen, J. K., et al. 2013, ApJ, 770, 123
Güdel, M., Lahuis, F., Briggs, K. R., et al. 2010, A\&A, 519, A113

Habing, H. J. 1968, Bull. Astr. Inst. Netherlands, 19, 421

Henning, T., Semenov, D., Guilloteau, S., et al. 2010, ApJ, 714, 1511

Herczeg, G. J., Karska, A., Bruderer, S., et al. 2012, A\&A, 540, A84

Isobe, T., \& Feigelson, E. D. 1990, BAAS, 22, 917

Isobe, T., Feigelson, E. D., \& Nelson, P. I. 1986, ApJ, 306, 490

Jonkheid, B., Dullemond, C. P., Hogerheijde, M. R., \& van Dishoeck, E. F. 2007, A\&A, 463, 203

Juhász, A., Bouwman, J., Henning, T., et al. 2010, ApJ, 721, 431

Kamp, I., \& Dullemond, C. P. 2004, ApJ, 615, 991

Karska, A., Herczeg, G. J., van Dishoeck, E. F., et al. 2013, A\&A, 552, A141

Kaufman, M. J., Wolfire, M. G., Hollenbach, D. J., \& Luhman, M. L. 1999, ApJ, 527,795

Kenyon, S. J., Gómez, M., \& Whitney, B. A. 2008, in Handbook of star forming regions, ed. B. Reipurth, 1, 405

Laraia, A. L., Gamache, R. R., Lamouroux, J., Gordon, I. E., \& Rothman, L. S. 2011, Icarus, 215, 391

Lavalley, M. P., Isobe, T., \& Feigelson, E. D. 1992, BAAS, 24, 839

Liseau, R., White, G. J., Larsson, B., et al. 1999, A\&A, 344, 342

Liskowsky, J. P., Brittain, S. D., Najita, J. R., et al. 2012, ApJ, 760, 153

Loinard, L., Torres, R. M., Mioduszewski, A. J., \& Rodríguez, L. F. 2008, ApJ, 675, L29

Lorenzetti, D. 2005, Space Sci. Rev., 119, 181

Lorenzetti, D., Tommasi, E., Giannini, T., et al. 1999, A\&A, 346, 604

Lorenzetti, D., Giannini, T., Nisini, B., et al. 2002, A\&A, 395, 637

Mandell, A. M., Mumma, M. J., Blake, G. A., et al. 2008, ApJ, 681, L25

Mandell, A. M., Bast, J., van Dishoeck, E. F., et al. 2012, ApJ, 747, 92

Meeus, G., Waters, L. B. F. M., Bouwman, J., et al. 2001, A\&A, 365, 476

Meeus, G., Montesinos, B., Mendigutía, I., et al. 2012, A\&A, 544, A78

Meeus, G., Salyk, C., Bruderer, S., et al. 2013, A\&A, 559, A84

Meijerink, R., Pontoppidan, K. M., Blake, G. A., Poelman, D. R., \& Dullemond, C. P. 2009, ApJ, 704, 1471

Müller, A., van den Ancker, M. E., Launhardt, R., et al. 2011, A\&A, 530, A85

Najita, J., Carr, J. S., \& Mathieu, R. D. 2003, ApJ, 589, 931

Najita, J. R., Ádámkovics, M., \& Glassgold, A. E. 2011, ApJ, 743, 147

Neuhäuser, R., \& Forbrich, J. 2008, in Handbook of star forming regions, ed. B. Reipurth, 2, 735

Pascucci, I., Apai, D., Luhman, K., et al. 2009, ApJ, 696, 143

Pilbratt, G. L., Riedinger, J. R., Passvogel, T., et al. 2010, A\&A, 518, L1

Podio, L., Kamp, I., Flower, D., et al. 2012, A\&A, 545, A44

Poglitsch, A., Waelkens, C., Geis, N., et al. 2010, A\&A, 518, L2

Pontoppidan, K. M., Salyk, C., Blake, G. A., et al. 2010, ApJ, 720, 887

Pontoppidan, K. M., Blake, G. A., \& Smette, A. 2011, ApJ, 733, 84

Röllig, M., Abel, N. P., Bell, T., et al. 2007, A\&A, 467, 187

Salyk, C., Pontoppidan, K. M., Blake, G. A., et al. 2008, ApJ, 676, L49

Salyk, C., Blake, G. A., Boogert, A. C. A., \& Brown, J. M. 2011a, ApJ, 743, 112

Salyk, C., Pontoppidan, K. M., Blake, G. A., Najita, J. R., \& Carr, J. S. 2011b, ApJ, 731, 130

Schöier, F. L., van der Tak, F. F. S., van Dishoeck, E. F., \& Black, J. H. 2005 , A\&A, 432, 369

Sternberg, A., \& Dalgarno, A. 1995, ApJS, 99, 565

Sturm, B., Bouwman, J., Henning, T., et al. 2010, A\&A, 518, L129

Thi, W.-F., Ménard, F., Meeus, G., et al. 2011, A\&A, 530, L2

Tilling, I., Woitke, P., Meeus, G., et al. 2012, A\&A, 538, A20

van der Plas, G., van den Ancker, M. E., Fedele, D., et al. 2008, A\&A, 485, 487

van der Plas, G., van den Ancker, M. E., Acke, B., et al. 2009, A\&A, 500, 1137

van der Tak, F. F. S., Black, J. H., Schöier, F. L., Jansen, D. J., \& van Dishoeck, E. F. 2007, A\&A, 468, 627

van Dishoeck, E. F. 2004, ARA\&A, 42, 119

van Kempen, T. A., Kristensen, L. E., Herczeg, G. J., et al. 2010, A\&A, 518, L121

van Leeuwen, F. 2007, A\&A, 474, 653

Waelkens, C., Waters, L. B. F. M., de Graauw, M. S., et al. 1996, A\&A, 315, L245

Wampfler, S. F., Herczeg, G. J., Bruderer, S., et al. 2010, A\&A, 521, L36

Wampfler, S. F., Bruderer, S., Karska, A., et al. 2013, A\&A, 552, A56

Woitke, P., Kamp, I., \& Thi, W. 2009, A\&A, 501, 383

Woitke, P., Pinte, C., Tilling, I., et al. 2010, MNRAS, 405, L26

Zhang, K., Pontoppidan, K. M., Salyk, C., \& Blake, G. A. 2013, ApJ, 766, 82

Pages 14 to 22 are available in the electronic edition of the journal at http://www . aanda. org 


\section{Appendix A: PACS Spectra}

Figure A.1 shows the PACS spectra of the T Tauri star AS 205 and of the Herbig Ae star HD 97048 between $62-190 \mu$ m. The main molecular and atomic transitions detected in the whole sample are shown. Figs. A.2 and A.3 show a portion of the PACS spectra of selected sources.
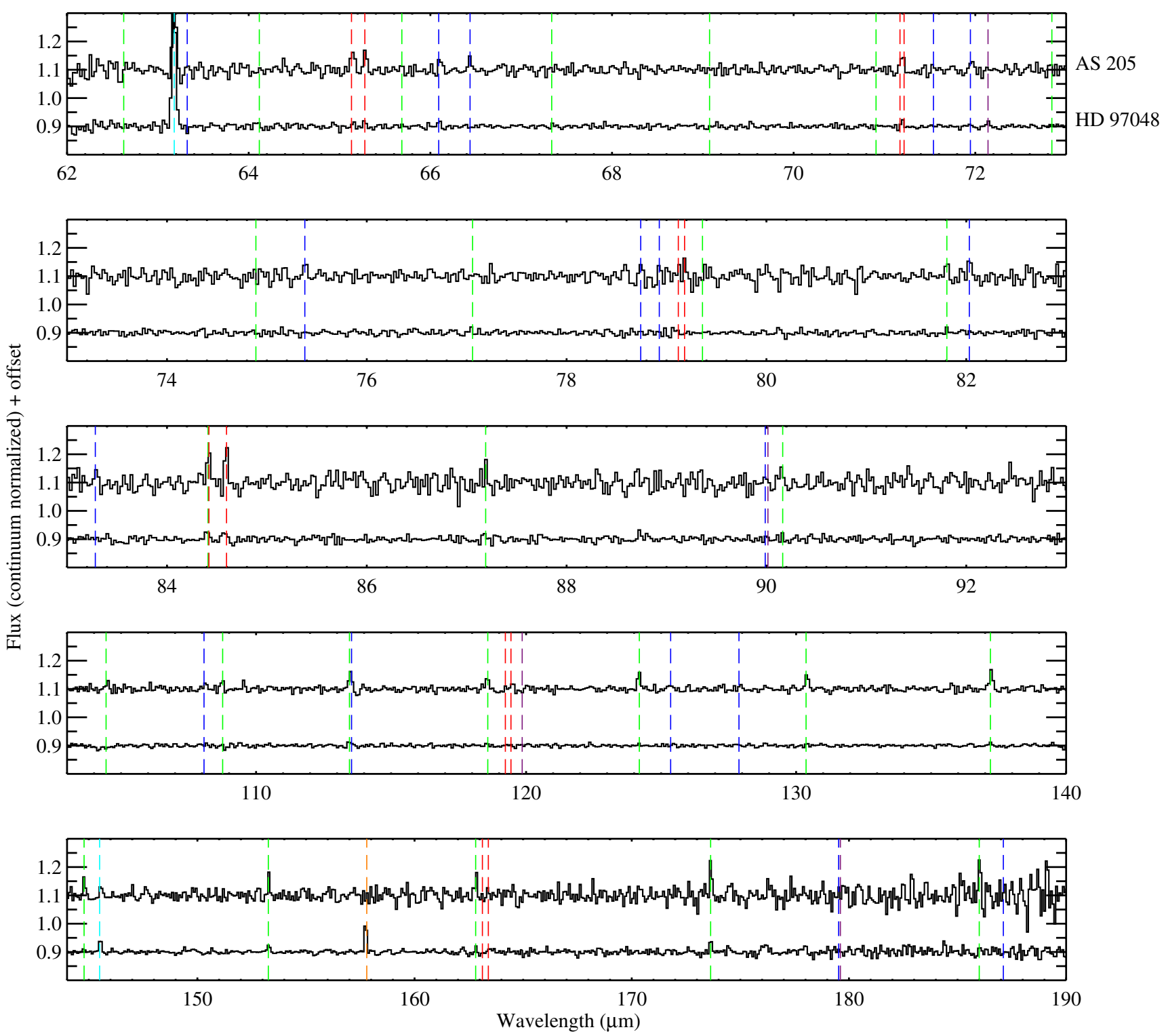

Fig. A.1. PACS spectrum of the T Tauri star AS 205 (top) and of the Herbig Ae star HD 97048 (bottom). The marks indicate the positions of [O I] (light blue), [C II] (orange), $\mathrm{CO}$ (green), $\mathrm{OH}$ (red), $\mathrm{H}_{2} \mathrm{O}$ (blue) and $\mathrm{CH}^{+}$(purple) lines. 
D. Fedele et al.: DIGIT survey of far-infrared lines from protoplanetary disks. I.

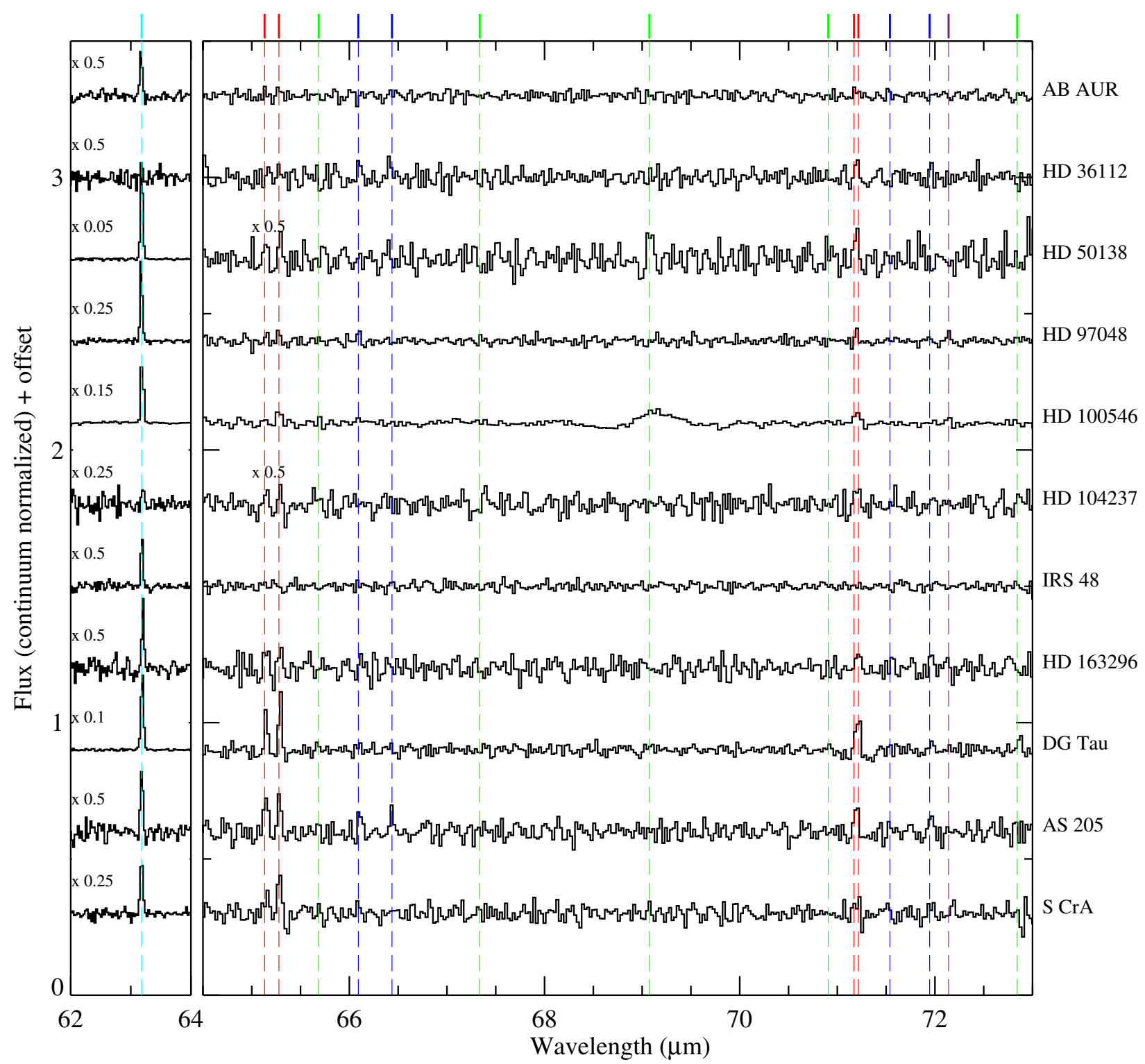

Fig. A.2. PACS spectra of a sub-sample of the program stars between $62-73 \mu \mathrm{m}$. Marks and colors as in Fig. A.1. The $69 \mu \mathrm{m}$ forsterite feature is present in the spectrum of HD 100546. 


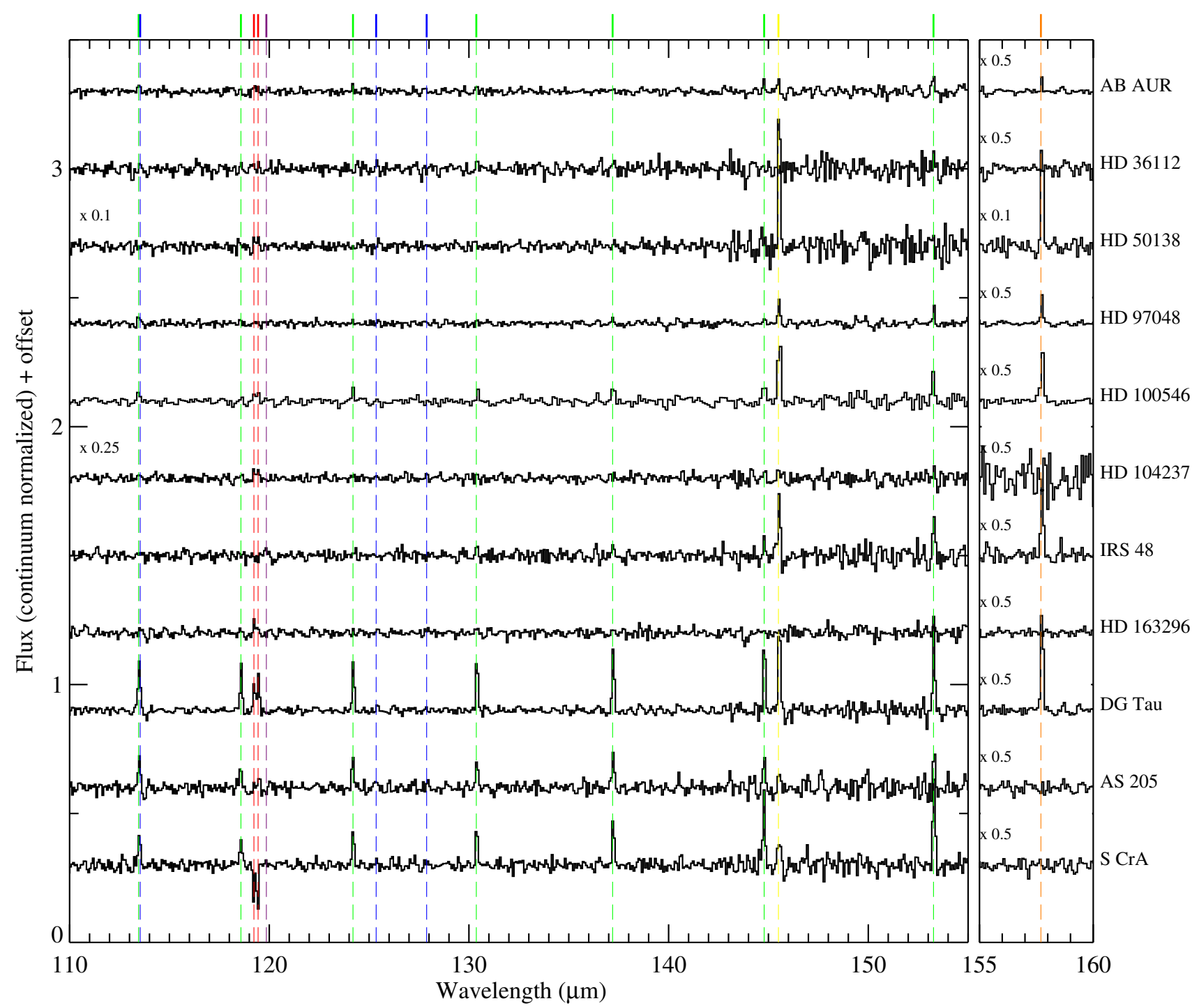

Fig. A.3. As Fig. A. 2 for the wavelength range $110-160 \mu \mathrm{m}$. 
D. Fedele et al.: DIGIT survey of far-infrared lines from protoplanetary disks. I.

\section{Appendix B: OH line fluxes and molecular data of selected species}

Table B.1 reports the line fluxes of the far-IR OH transitions. The line flux uncertainties correspond to the $1 \sigma$ error. For non-detection the $3 \sigma$ upper limit is reported. Table B.2 reports the atomic and molecular data of the transitions detected in this paper. Molecular data are taken from the LAMDA database (Schöier et al. 2005).

Table B.1. OH line fluxes.

\begin{tabular}{|c|c|c|c|c|c|c|c|c|}
\hline & \multicolumn{6}{|c|}{${ }^{2} \Pi_{3 / 2}$} & \multicolumn{2}{|c|}{${ }^{2} \Pi_{1 / 2}-{ }^{2} \Pi_{3 / 2}$} \\
\hline & $\begin{array}{c}9 / 2^{-}-7 / 2^{+} \\
65.13 \mu \mathrm{m} \\
\end{array}$ & $\begin{array}{c}9 / 2^{+}-7 / 2^{-} \\
65.28 \mu \mathrm{m} \\
\end{array}$ & $\begin{array}{c}7 / 2^{-}-5 / 2^{+} \\
84.42 \mu \mathrm{m} \\
\end{array}$ & $\begin{array}{c}7 / 2^{+}-5 / 2^{-} \\
84.59 \mu \mathrm{m} \\
\end{array}$ & $\begin{array}{l}5 / 2^{-}-3 / 2^{+} \\
119.23 \mu \mathrm{m} \\
\end{array}$ & $\begin{array}{c}5 / 2^{+}-3 / 2^{-} \\
119.44 \mu \mathrm{m} \\
\end{array}$ & $\begin{array}{c}1 / 2^{-}-3 / 2^{+} \\
79.11 \mu \mathrm{m}\end{array}$ & $\begin{array}{c}1 / 2^{+}-3 / 2^{-} \\
79.18 \mu \mathrm{m}\end{array}$ \\
\hline AB Aur & $7.0 \pm 2.0$ & $12.5 \pm 2.0$ & $10.1 \pm 1.5$ & $10.1 \pm 1.5$ & $2.8 \pm 1.4$ & $3.1 \pm 1.4$ & $<9.0$ & $<9.0$ \\
\hline HD 36112 & $2.2 \pm 0.5$ & $2.9 \pm 0.5$ & $2.5 \pm 0.8$ & $2.6 \pm 0.8$ & $0.49 \pm 0.20$ & $0.52 \pm 0.12$ & $<3.4$ & $<3.4$ \\
\hline HD 38120 & $<3.5$ & $<3.5$ & $<2.8$ & $<2.8$ & $<0.9$ & $<0.9$ & $<2.7$ & $<2.7$ \\
\hline HD 50138 & $4.0 \pm 0.8$ & $4.1 \pm 0.8$ & $a$ & $1.9 \pm 0.6$ & $1.0 \pm 0.2$ & $1.1 \pm 0.2$ & $<4.0$ & $<4.0$ \\
\hline HD 97048 & $4.9 \pm 1.0$ & $5.7 \pm 1.0$ & $6.8 \pm 1.0$ & $6.8 \pm 1.0$ & $<2.4$ & $<2.4$ & $<4.0$ & $<4.0$ \\
\hline HD 100453 & $<3.4$ & $<3.4$ & $<3.0$ & $<3.0$ & $<1.3$ & $<1.3$ & $<2.0$ & $<2.0$ \\
\hline HD 100546 & $13.6 \pm 0.4$ & $19.9 \pm 3.3$ & $a$ & $13.8 \pm 2.0$ & $4.2 \pm 0.9$ & $4.2 \pm 0.9$ & $<6.0$ & $<6.0$ \\
\hline HD 104237 & $3.0 \pm 0.5$ & $3.0 \pm 0.5$ & $3.1 \pm 0.5$ & $3.1 \pm 0.5$ & $1.2 \pm 0.3$ & $1.2 \pm 0.3$ & $<3.6$ & $<3.6$ \\
\hline HD 135344 B & $<4.0$ & $<4.0$ & $<3.8$ & $<3.8$ & $0.67 \pm 0.19$ & $0.71 \pm 0.16$ & $<3.0$ & $<3.0$ \\
\hline HD 139614 & $<4.6$ & $<4.6$ & $<3.6$ & $<3.6$ & $<1.2$ & $<1.2$ & $<3.0$ & $<3.0$ \\
\hline HD 142527 & $<9.0$ & $<9.0$ & $6.6 \pm 1.1$ & $5.0 \pm 2.0$ & $<4.0$ & $<4.0$ & $<4.0$ & $<4.0$ \\
\hline HD 144668 & $<4.5$ & $<4.5$ & $<4.8$ & $<4.8$ & $<0.8$ & $<0.8$ & $<4.0$ & $<4.0$ \\
\hline IRS 48 & $<9.6$ & $<9.6$ & $<4.0$ & $<4.0$ & $<1.2$ & $<1.2$ & $<3.0$ & $<3.0$ \\
\hline HD 163296 & $5.3 \pm 0.8$ & $4.5 \pm 0.8$ & $2.8 \pm 0.5$ & $2.7 \pm 0.5$ & $1.3 \pm 0.2$ & $0.74 \pm 0.23$ & $<3.0$ & $<3.0$ \\
\hline HD 169142 & $<8.4$ & $<8.4$ & $<7.9$ & $<7.9$ & $<2.4$ & $<2.4$ & $<4.0$ & $<4.0$ \\
\hline HD 179218 & $<3.2$ & $<3.2$ & $<2.7$ & $<2.7$ & $<1.1$ & $<1.1$ & $<2.0$ & $<2.0$ \\
\hline DG Tau & $5.9 \pm 0.7$ & $8.9 \pm 0.7$ & $10.6 \pm 0.7$ & $10.4 \pm 0.7$ & $2.6 \pm 0.4$ & $3.8 \pm 0.4$ & $4.7 \pm 0.8$ & $4.7 \pm 0.8$ \\
\hline AS 205 & $6.5 \pm 1.0$ & $6.4 \pm 1.0$ & $4.8 \pm 0.8$ & $4.7 \pm 0.8$ & $0.8 \pm 0.3$ & $1.3 \pm 0.3$ & $2.2 \pm 0.8$ & $2.5 \pm 0.8$ \\
\hline SR 21 & $<3.8$ & $<3.8$ & $<3.9$ & $<3.8$ & $<1.3$ & $<1.3$ & $<3.5$ & $<3.5$ \\
\hline \multirow[t]{3}{*}{ S CrA } & $5.0 \pm 0.6$ & $5.8 \pm 0.6$ & $4.5 \pm 0.7$ & $5.1 \pm 0.7$ & absorption & absorption & $2.9 \pm 0.8$ & $<4.5$ \\
\hline & \multicolumn{6}{|c|}{${ }^{2} \Pi_{1 / 2}$} & & \\
\hline & $\begin{array}{c}9 / 2^{-}-7 / 2^{+} \\
55.89 \mu \mathrm{m} \\
\end{array}$ & $\begin{array}{c}9 / 2^{+}-7 / 2^{-} \\
55.95 \mu \mathrm{m}\end{array}$ & $\begin{array}{c}7 / 2^{-}-5 / 2^{+} \\
71.17 \mu \mathrm{m} \\
\end{array}$ & $\begin{array}{c}7 / 2^{+}-5 / 2^{-} \\
71.21 \mu \mathrm{m}\end{array}$ & $\begin{array}{c}3 / 2^{+}-1 / 2^{-} \\
163.12 \mu \mathrm{m}\end{array}$ & $\begin{array}{c}3 / 2^{-} 1 / 2^{+} \\
163.40 \mu \mathrm{m}\end{array}$ & & \\
\hline AB Aur & $<22.4$ & $<22.4$ & $4.5 \pm 0.6$ & $4.5 \pm 0.6$ & $<3.3$ & $<3.3$ & & \\
\hline HD 36112 & $<7.6$ & $<7.6$ & $1.2 \pm 0.2$ & $1.2 \pm 0.3$ & $<1.5$ & $<1.5$ & & \\
\hline HD 38120 & $<5.6$ & $<5.6$ & $<2.3$ & $<2.3$ & $<1.3$ & $<1.3$ & & \\
\hline HD 50138 & $<8.0$ & $<8.0$ & $2.0 \pm 0.4$ & $2.0 \pm 0.4$ & $<1.8$ & $<1.8$ & & \\
\hline HD 97048 & $3.0 \pm 0.8$ & $2.4 \pm 0.8$ & $1.8 \pm 0.4$ & $1.8 \pm 0.4$ & $<2.5$ & $<2.5$ & & \\
\hline HD 100453 & $<5.5$ & $<5.5$ & $<3.0$ & $<3.0$ & $<1.4$ & $<1.4$ & & \\
\hline HD 100546 & $<16.0$ & $<16.0$ & $8.0 \pm 1.4$ & $8.0 \pm 1.4$ & $<3.7$ & $<3.7$ & & \\
\hline HD 104237 & $2.4 \pm 0.8$ & $2.7 \pm 0.8$ & $1.4 \pm 0.3$ & $1.4 \pm 0.3$ & $<1.7$ & $<1.7$ & & \\
\hline HD 135344 B & $<8.2$ & $<8.2$ & $<2.7$ & $<2.7$ & $<1.6$ & $<1.6$ & & \\
\hline HD 139614 & $<8.5$ & $<8.5$ & $<2.9$ & $<2.9$ & $<1.6$ & $<1.6$ & & \\
\hline HD 142527 & $<13.0$ & $<13.0$ & $<6.3$ & $<6.3$ & $<2.8$ & $<2.8$ & & \\
\hline HD 144668 & $<7.8$ & $<7.8$ & $<2.9$ & $<2.9$ & $<2.3$ & $<2.3$ & & \\
\hline IRS 48 & $<8.3$ & $<8.3$ & $<2.9$ & $<2.9$ & $<1.6$ & $<1.7$ & & \\
\hline HD 163296 & $4.8 \pm 1.0$ & $6.0 \pm 1.0$ & $<1.8$ & $2.9 \pm 0.3$ & $<1.4$ & $<1.4$ & & \\
\hline HD 169142 & $<13.5$ & $<13.5$ & $<5.8$ & $<5.8$ & $<2.8$ & $<2.8$ & & \\
\hline HD 179218 & $<7.0$ & $<7.0$ & $<2.2$ & $<2.2$ & $<1.0$ & $<1.0$ & & \\
\hline DG Tau & $5.0 \pm 1.0$ & $5.0 \pm 1.0$ & $4.0 \pm 1.0$ & $4.0 \pm 1.0$ & $0.9 \pm 0.4$ & $1.3 \pm 0.4$ & & \\
\hline AS 205 & $3.8 \pm 1.2$ & $5.6 \pm 1.2$ & $2.1 \pm 0.6$ & $2.1 \pm 0.6$ & $<1.5$ & $<1.5$ & & \\
\hline SR 21 & $<5.8$ & $<5.8$ & $<3.2$ & $<3.2$ & $<1.5$ & $<1.5$ & & \\
\hline $\mathrm{S} \mathrm{CrA}$ & $<6.0$ & $<6.0$ & $1.6 \pm 0.6$ & $1.9 \pm 0.6$ & $<1.6$ & $<1.6$ & & \\
\hline
\end{tabular}

Notes. Units and upper limits as in Table 3. ${ }^{(a)}$ Blended with CO $J=31-30$. 
Table B.2. Atomic and molecular data of the far-IR detected transitions.

\begin{tabular}{|c|c|c|c|c|}
\hline Species & Transition & $\begin{array}{c}\lambda \\
{[\mu \mathrm{m}]}\end{array}$ & $\begin{array}{c}E_{u} \\
{[\mathrm{~K}]} \\
\end{array}$ & $\begin{array}{c}\log \left(A_{\mathrm{ul}}\right) \\
{\left[\mathrm{s}^{-1}\right]}\end{array}$ \\
\hline $\mathrm{OH}$ & ${ }^{2} \Pi_{1 / 2} 9 / 2^{+}-7 / 2^{-}$ & 55.891 & 875 & 0.34 \\
\hline $\mathrm{OH}$ & ${ }^{2} \Pi_{1 / 2} 9 / 2^{-}-7 / 2^{+}$ & 55.949 & 875 & 0.34 \\
\hline $\mathrm{OH}$ & ${ }^{2} \Pi_{3 / 2} 9 / 2^{-}-7 / 2^{+}$ & 65.131 & 512 & 0.11 \\
\hline $\mathrm{OH}$ & ${ }^{2} \Pi_{3 / 2} 9 / 2^{+}-7 / 2^{-}$ & 65.278 & 510 & 0.10 \\
\hline $\mathrm{OH}$ & ${ }^{2} \Pi_{1 / 2} 7 / 2^{-}-5 / 2^{+}$ & 71.170 & 617 & 0.01 \\
\hline $\mathrm{OH}$ & ${ }^{2} \Pi_{1 / 2} 7 / 2^{+}-5 / 2^{-}$ & 71.215 & 617 & 0.01 \\
\hline $\mathrm{OH}$ & ${ }^{2} \Pi_{1 / 2}-{ }^{2} \Pi_{3 / 2} J=1 / 2^{-}-3 / 2^{+}$ & 79.115 & 181 & -1.44 \\
\hline $\mathrm{OH}$ & ${ }^{2} \Pi_{1 / 2}-{ }^{2} \Pi_{3 / 2} J=1 / 2^{+}-3 / 2^{-}$ & 79.178 & 181 & -1.44 \\
\hline $\mathrm{OH}$ & ${ }^{2} \Pi_{3 / 2} 7 / 2^{-}-5 / 2^{+}$ & 84.420 & 291 & -0.28 \\
\hline $\mathrm{OH}$ & ${ }^{2} \Pi_{3 / 2} 7 / 2^{+}-5 / 2^{-}$ & 84.596 & 290 & -0.28 \\
\hline $\mathrm{OH}$ & ${ }^{2} \Pi_{3 / 2} 5 / 2^{-}-3 / 2^{+}$ & 119.233 & 120 & -0.86 \\
\hline $\mathrm{OH}$ & ${ }^{2} \Pi_{3 / 2} 5 / 2^{+}-3 / 2^{-}$ & 119.441 & 120 & -0.86 \\
\hline $\mathrm{OH}$ & ${ }^{2} \Pi_{1 / 2} 3 / 2^{+}-1 / 2^{-}$ & 163.120 & 270 & -1.190 \\
\hline $\mathrm{OH}$ & ${ }^{2} \Pi_{1 / 2} 3 / 2^{-}-1 / 2^{+}$ & 163.410 & 270 & -1.190 \\
\hline $\mathrm{CH}^{+}$ & $J=6-5$ & 60.248 & 839 & 0.27 \\
\hline $\mathrm{CH}^{+}$ & $J=5-4$ & 72.141 & 600 & 0.03 \\
\hline $\mathrm{CH}^{+}$ & $J=4-3$ & 90.017 & 400 & -0.26 \\
\hline $\mathrm{CH}^{+}$ & $J=3-2$ & 119.858 & 240 & -0.66 \\
\hline $\mathrm{CH}^{+}$ & $J=2-1$ & 179.605 & 120 & -1.21 \\
\hline $\mathrm{o}-\mathrm{H}_{2} \mathrm{O}$ & $4_{32}-3_{21}$ & 58.70 & 550 & 0.14 \\
\hline $\mathrm{p}-\mathrm{H}_{2} \mathrm{O}$ & $7_{26}-6_{15}$ & 59.99 & 1020 & 0.13 \\
\hline $\mathrm{O}-\mathrm{H}_{2} \mathrm{O}$ & $8_{18}-7_{07}$ & 63.32 & 1070 & 0.24 \\
\hline $\mathrm{o}-\mathrm{H}_{2} \mathrm{O}$ & $7_{16}-6_{25}$ & 66.09 & 1013 & -0.02 \\
\hline $\mathrm{o}-\mathrm{H}_{2} \mathrm{O}$ & $3_{30}-2_{21}$ & 66.44 & 410 & 0.09 \\
\hline $\mathrm{o}-\mathrm{H}_{2} \mathrm{O}$ & $7_{07}-6_{16}$ & 71.95 & 843 & 0.06 \\
\hline $\mathrm{o}-\mathrm{H}_{2} \mathrm{O}$ & $3_{21}-2_{12}$ & 75.38 & 305 & -0.48 \\
\hline $\mathrm{o}-\mathrm{H}_{2} \mathrm{O}$ & $4_{23}-3_{12}$ & 78.74 & 432 & -0.32 \\
\hline $\mathrm{p}-\mathrm{H}_{2} \mathrm{O}$ & $6_{15}-5_{24}$ & 78.93 & 781 & -0.34 \\
\hline $\mathrm{O}-\mathrm{H}_{2} \mathrm{O}$ & $6_{16}-5_{05}$ & 82.03 & 643 & 0.06 \\
\hline $\mathrm{p}-\mathrm{H}_{2} \mathrm{O}$ & $6_{06}-5_{15}$ & 83.28 & 642 & -0.15 \\
\hline $\mathrm{O}-\mathrm{H}_{2} \mathrm{O}$ & $2_{21}-1_{10}$ & 108.07 & 194 & -0.59 \\
\hline $\mathrm{o}-\mathrm{H}_{2} \mathrm{O}$ & $4_{14}-3_{03}$ & 113.54 & 323 & -0.61 \\
\hline $\mathrm{o}-\mathrm{H}_{2} \mathrm{O}$ & $2_{12}-1_{01}$ & 179.53 & 114 & -1.25 \\
\hline $\mathrm{p}-\mathrm{H}_{2} \mathrm{O}$ & $4_{13}-4_{04}$ & 187.11 & 396 & -1.43 \\
\hline [O I] & ${ }^{3} P_{1}-{ }^{3} P_{2}$ & 63.185 & 228 & -4.05 \\
\hline [O I] & ${ }^{3} P_{0}-{ }^{3} P_{2}$ & 145.535 & 327 & -4.75 \\
\hline$[\mathrm{C}$ II $]$ & ${ }^{2} P_{3 / 2}-{ }^{2} P_{1 / 2}$ & 157.680 & 91 & -5.64 \\
\hline
\end{tabular}


D. Fedele et al.: DIGIT survey of far-infrared lines from protoplanetary disks. I.

\section{Appendix C: Effects of non-LTE excitation of $\mathrm{OH}$ far-IR}
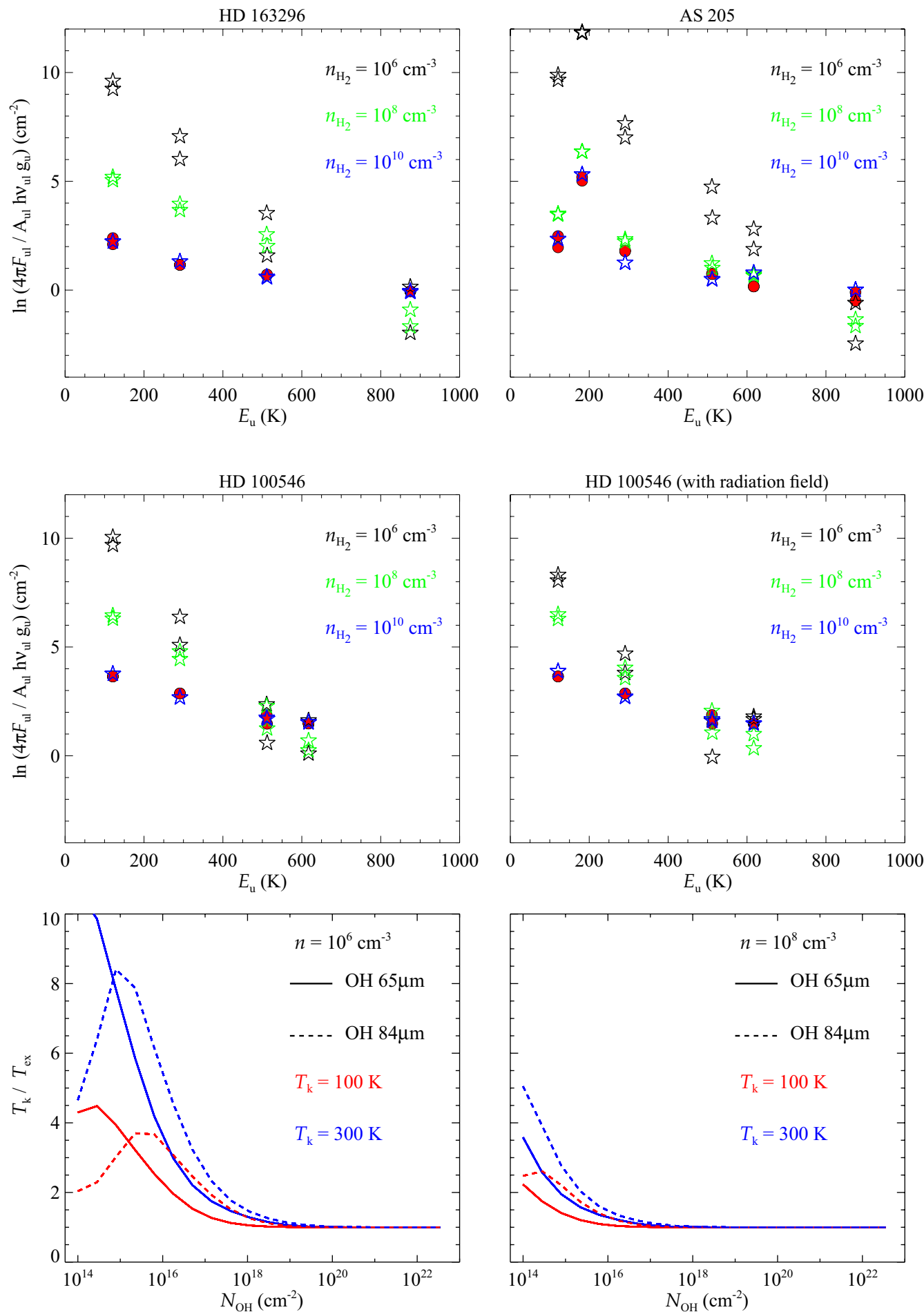

Fig. C.1. Results of the non-LTE simulations with RADEX. (Top) OH rotational diagram of HD 163296 and AS 205 and non-LTE model predictions: three different models are shown for different values of the gas density and a temperature of $400 \mathrm{~K}$ and $200 \mathrm{~K}$ for HD 163296 and AS 205 , respectively. Only models with $n \geq 10^{10} \mathrm{~cm}^{-3}$ can reproduce the observations. (Middle) OH rotational diagram of HD 100546 and non-LTE model predictions $\left(N=2 \times 10^{14} \mathrm{~cm}^{-2}, T=200 \mathrm{~K}\right)$ in the case in which the infrared radiation field is included in the RADEX simulation to test the effect of infrared pumping: in both cases (with and without radiation field) high gas densities are needed to reproduce the observed rotational diagram. (Bottom) Ratio of $T_{\mathrm{k}}$ to $T_{\mathrm{ex}}$ for $2 \mathrm{OH}$ transitions as a function of $N_{\mathrm{OH}}$ in two low gas density cases. Even in the low gas density cases, the $\mathrm{OH}$ rotational levels are in LTE $\left(T_{\mathrm{k}}=T_{\mathrm{ex}}\right)$ for large values of $N_{\mathrm{OH}}$ when the lines are optically thick.

$\mathrm{OH}$ lines studied here have large critical densities $\left(n_{\text {crit }} \sim\right.$ $10^{9}-10^{10} \mathrm{~cm}^{-3}$ ) and non-LTE excitation may be important if the gas density is not high enough to thermalize the $\mathrm{OH}$ molecules.
To test the assumption of LTE we fit the observed rotational diagram using the non-LTE code RADEX (van der Tak et al. 2007). We used the same fitting procedure as for the LTE 
case and we repeated the analysis for different values of the gas $\left(\mathrm{H}_{2}\right.$, collision partner) density $\left(n=10^{6}, 10^{8}, 10^{10} \mathrm{~cm}^{-3}\right)$. Figure C.1 shows the $\mathrm{OH}$ rotational diagram for two test cases: the Herbig Ae HD 163296 and the T Tauri AS 205 disks. We reproduced the slab model using the best-fit parameters found in the LTE case with $N_{\mathrm{OH}}=10^{15} \mathrm{~cm}^{-2}$ and $T_{\mathrm{K}}=400 \mathrm{~K}$ for HD 163296 and $N_{\mathrm{OH}}=8 \times 10^{15} \mathrm{~cm}^{-2}$ and $T_{\mathrm{K}}=200 \mathrm{~K}$ for AS 205 (Table 6). The non-LTE model predictions are plotted in Fig. C. 1 with different colors for the three values of $n_{\mathrm{H}_{2}}$. For low $n_{\mathrm{H}_{2}}$ values $\left(\leq 10^{8} \mathrm{~cm}^{-3}\right)$ the model fails to reproduce the observed rotational diagram. The gas density must be $n_{\mathrm{H}_{2}} \geq 10^{10} \mathrm{~cm}^{-3}$ in order to fit the observations. Thus, the $\mathrm{OH}$ rotational lines emerge from an high density region where the $\mathrm{OH}$ molecules are thermalized and the rotational levels are in LTE.

Infrared pumping can be relevant for the excitation of $\mathrm{OH}$ molecules. To test the effects of line pumping we run a grid of RADEX models for HD 100546 providing also the infrared radiation field (between $20 \mu \mathrm{m}-3 \mathrm{~mm}$ ) in the input parameters.

The radiation field is taken from the full disk thermochemical model of Bruderer et al. (2012) who computed the radiation field at each position of the disk for different wavelengths. The radiation field is stronger in the inner region of the disk $(r<20 \mathrm{AU})$. As input to RADEX we considered the value of the infrared radiation field at a distance of $r=20 \mathrm{AU}$ and height above the midplane $z=4 \mathrm{AU}(z / r=0.2)$. At larger radii and height (where the far-IR OH lines originate) the radiation field is always fainter. Figure C.1 shows the $\mathrm{OH}$ rotational diagram of HD 100546 (middle row) and the RADEX predictions without (left) and with (right) infrared radiation field. The line flux ratios vary in the presence of infrared pumping, but even in this case high gas density $\left(\geq 10^{10} \mathrm{~cm}^{-3}\right)$ is needed to reproduce the observed rotational diagram.

The non-LTE simulations also show that for large values of the column density $\left(N_{\mathrm{OH}} \geq 10^{18} \mathrm{~cm}^{-2}\right)$ the $\mathrm{OH}$ rotational lines are in LTE even at gas densities $\leq 10^{8} \mathrm{~cm}^{-3}$ (Fig. C.1, bottom).

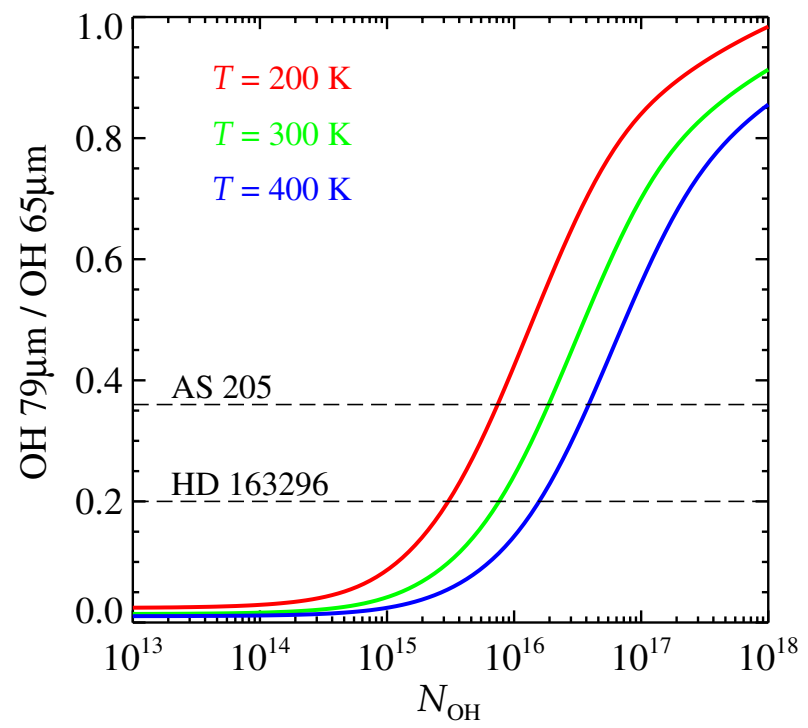

Fig. C.2. Ratio of the $\mathrm{OH} 79 \mu \mathrm{m}$ to the $\mathrm{OH} 65 \mu \mathrm{m}$ lines from the LTE calculation at 3 different temperatures. The ratio increases rapidly with column density. The dashed lines indicate the observed ratio for AS 205 and HD 163296 ( $1 \sigma$ upper limit).

This is due to line opacity which traps the photons and helps to thermalize the gas. However, we can exclude this scenario for most of the sources based on the non-detection of the intraladder transitions at $79 \mu \mathrm{m}$. These transitions are indeed very sensitive to line opacity and the lines are easily detected for $N_{\mathrm{OH}} \gtrsim 10^{16} \mathrm{~cm}^{-2}$, as in the case of DG Tau and AS 205. This is shown in Fig. C. 2 where the ratio of the $\mathrm{OH} 79 \mu \mathrm{m}$ to the $\mathrm{OH} 65 \mu \mathrm{m}$ lines is shown in the LTE case for different temperatures. In order for the intra-ladder lines to be detected high column density is needed. 


\section{Appendix D: [O I], [C II] spatial extent}

This section describes an analysis of the atomic lines aiming at addressing the spatial extent of the line. The Herschel/PACS PSF varies substantially from $50 \mu \mathrm{m}$ to $200 \mu \mathrm{m}$. As a consequence the amount of flux in the central spaxel varies from $\sim 70 \%$ at $60 \mu \mathrm{m}$ to $55 \%$ at $160 \mu \mathrm{m}$. For this reason, line emission can be detected outside the central spaxel (especially in the red part $>100 \mu \mathrm{m}$ ). To check whether a line is spatially extended we compute the equivalent width $(W)$ and integrated continuum $\left(F_{c}\right)$ next to the line and check the relative spatial distribution. If the line emission is co-spatial to the continuum emission, then the spatial distribution of the equivalent width will be equal to that of the integrated continuum $\left(F_{\mathrm{c}}\right)$ (same PSF). In particular, the distribution of $F_{\mathrm{c}}$ corresponds to the PSF at the given wavelength (assuming that the continuum emission is not spatially resolved).

For the $[\mathrm{O} \mathrm{I}] 63 \mu \mathrm{m}$ line, $F_{\mathrm{C}}$ is measured integrating the spectrum between $64.0-64.5 \mu \mathrm{m}$ and the equivalent width is measured integrating the spectrum between $63.08-63.30 \mu \mathrm{m}$. The only source where off-source oxygen excess emission is DG Tau. Figure D. 1 shows the [O I] $63 \mu \mathrm{m}$ spectral map of DG Tau: the (blue) dashed contours show the distribution of the spectral continuum and the sub-panels shows the [O I] $63 \mu \mathrm{m}$ spectrum in each spaxel. While the continuum is compact and centered on the central spaxel, the line emission shows an excess emission outside the central spaxel. The maximum excess is measured southward of the central source in agreement with the outflow position.
For the [C II] line the line is integrated between 157.530 and $157.970 \mu \mathrm{m}$ and the continuum flux between 158.5 and $162 \mu \mathrm{m}$. Figures D.1 and D.2 show the line spectral map for different sources. The spectral map shows the spectrum (continuum subtracted) in each spaxel. All the sources where [C II] emission is detected show excess line emission outside the central spaxel. The most clear cases are HD 38120, IRS 48 and DG Tau. This pattern is the result of extended line emission. In the case of AB Aur and HD 97048 the object is mis-pointed and the spatial distribution of the continuum emission deviates from the PSF. Nevertheless, also in these two cases the line emission is not co-spatial with the continuum emission and proves a spatially extended line emission. The case of HD 50138 is less clear.

\section{D.1. On-source [C II] line flux}

To estimate the maximum [C II] emission associated with the protoplanetary disk the extended emission needs to be subtracted. To do this the [C II] line flux (integrated between 157.60 and $157.98 \mu \mathrm{m}$ ) in each of the 9 central spaxels is calculated. Then the extended emission is determined as the average of the line flux measured in the 8 neighboring spaxels (around the central one) and subtracted from the value measured in the central spaxel. The result is reported in Table 3. In this way, the large scale $(>9.4)$ [C II] emission is approximately removed. The value of the [C II] flux derived by this method must be considered an upper limit to the [C II] emission arising from the disk as extended emission from a compact remnant envelope may still

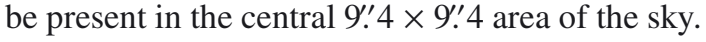
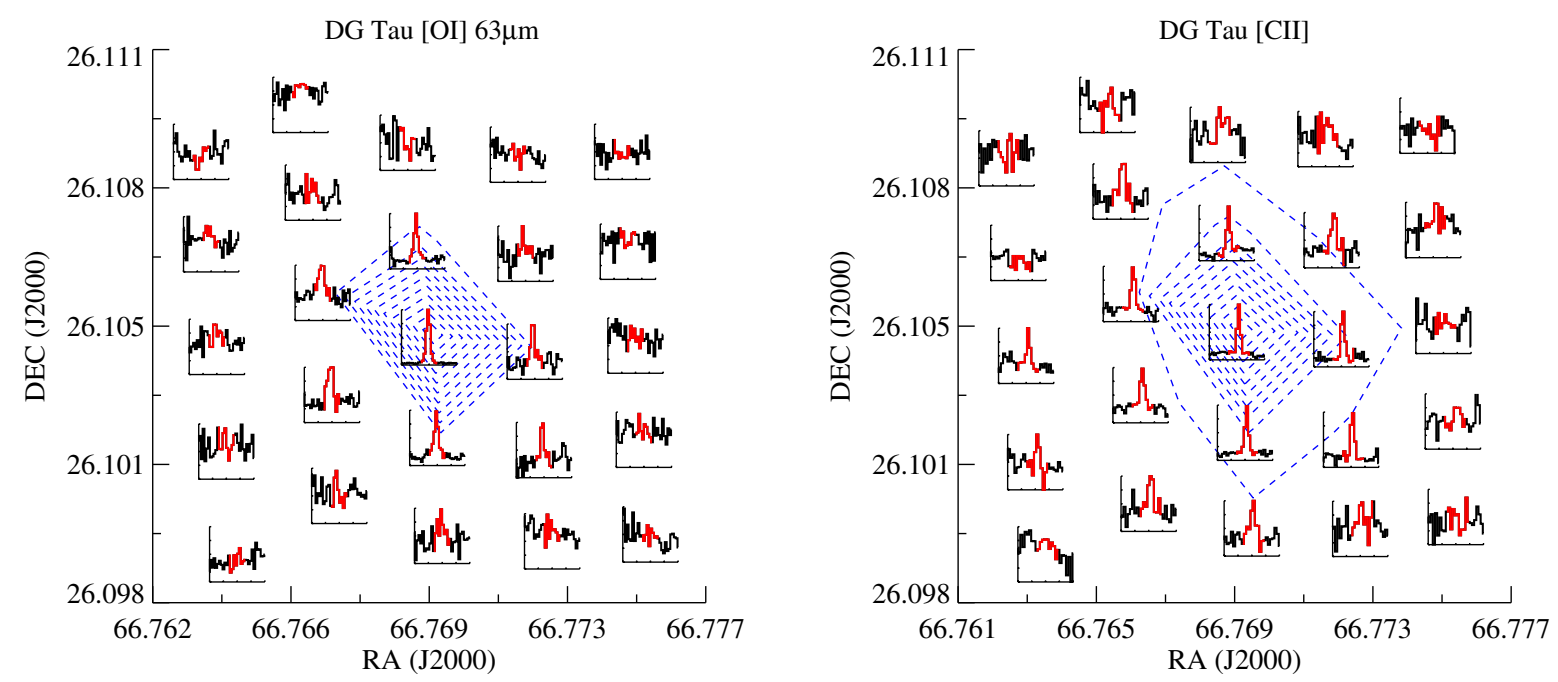

Fig. D.1. [O I] $63 \mu \mathrm{m}$ and [C II] spectral map in DG Tau showing the spatially extended line emission. The contours represent the spectral continuum measured in the vicinity of the line, the last contour level corresponds to $10 \%$ of the continuum peak. The sub-panels show the line spectrum measured in each spaxel. 

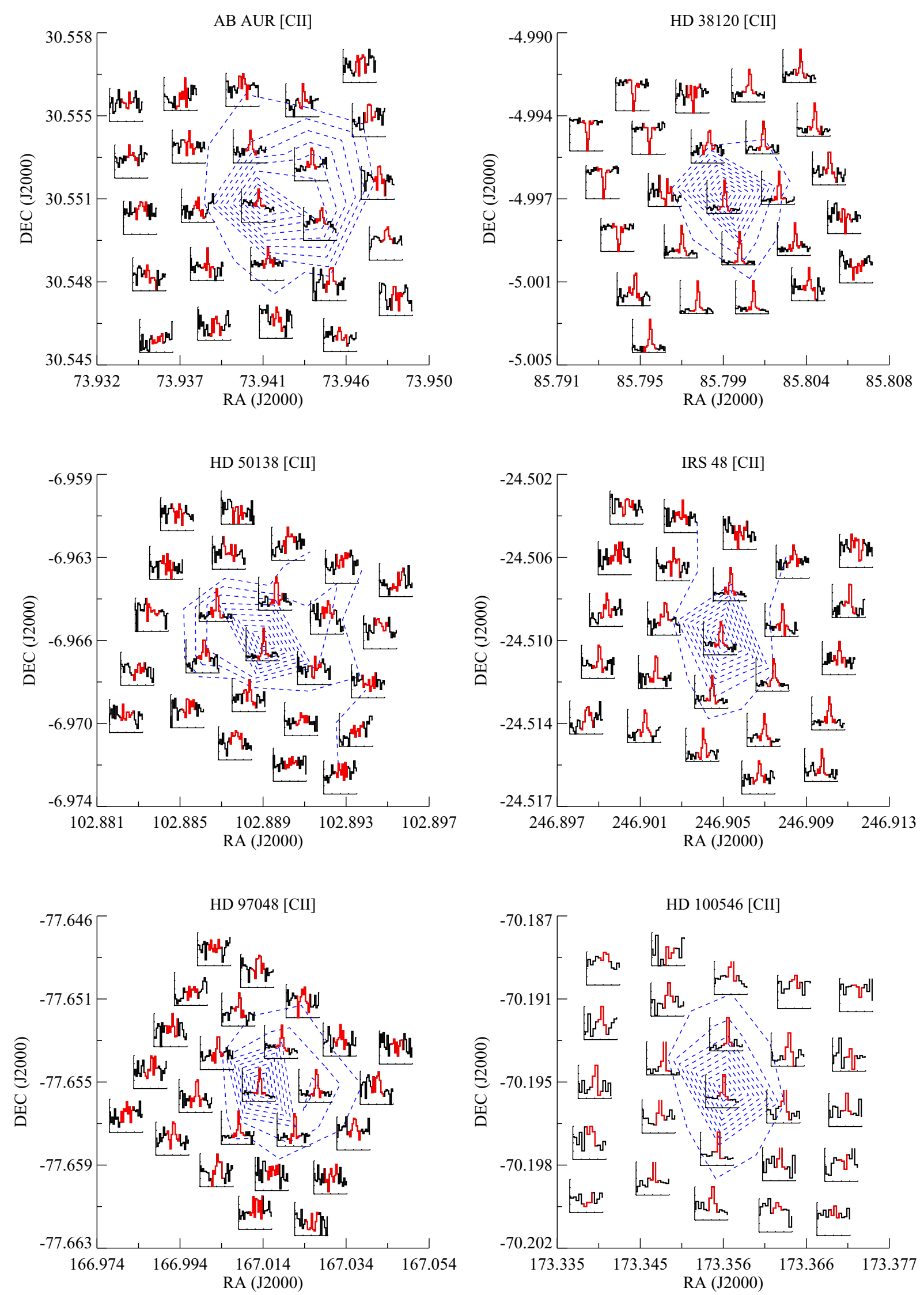

Fig. D.2. Same as Fig. D.1 for [C II] emission in Herbig AeBe sources. 